TRANSACTIONS OF THE

AMERICAN MATHEMATICAL SOCIETY

Volume 365, Number 1, January 2013, Pages 459-490

S 0002-9947(2012)05635-2

Article electronically published on August 24, 2012

\title{
SUM FORMULAS FOR LOCAL GROMOV-WITTEN INVARIANTS OF SPIN CURVES
}

\author{
JUNHO LEE
}

\begin{abstract}
Holomorphic 2-forms on Kähler surfaces lead to "local GromovWitten invariants" of spin curves. This paper shows how to derive sum formulas for such local GW invariants from the sum formula for GW invariants of certain ruled surfaces. These sum formulas also verify the Maulik-Pandharipande formulas that were recently proved by Kiem and Li.
\end{abstract}

Let $X$ be a Kähler surface with a holomorphic 2 -form $\alpha$. The real part of $\alpha$, also denoted by $\alpha$, then induces an almost complex structure on $X$ :

$$
J_{\alpha}=\left(I d+J K_{\alpha}\right)^{-1} J\left(I d+J K_{\alpha}\right) .
$$

Here $J$ is the Kähler structure on $X$ and $K_{\alpha}$ is the endomorphism of $T X$ defined by the formula $\left\langle u, K_{\alpha} v\right\rangle=\alpha(u, v)$, where $\langle$,$\rangle is the Kähler metric on X$. The almost complex structure $J_{\alpha}$ satisfies a remarkable Image Localization Property:

- if $f$ is a $J_{\alpha}$-holomorphic map into $X$ that represents a non-zero $(1,1)$ class, then the image of $f$ lies in the zero set $D$ of the holomorphic 2-form $\alpha$.

For simplicity, assume $X$ is a (minimal) surface of general type and $D$ is smooth. The normal bundle $N$ to $D$ is then a theta characteristic on $D$, i.e., $N$ is a square root of the canonical bundle of $D$. The pair $(D, N)$ is called a spin curve of genus $h$ where $h$ is the genus of $D$. The total space $N_{D}$ of $N$ has a tautological holomorphic 2 -form $\alpha$ that induces, by the same manner as in (0.1), an almost complex structure $J_{\alpha}$ on $N_{D}$ satisfying the image localization property, namely

$$
\overline{\mathcal{M}}_{g, n}\left(N_{D}, d[D], J_{\alpha}\right)=\overline{\mathcal{M}}_{g, n}(D, d) .
$$

Consequently, the moduli space of $J_{\alpha}$-holomorphic maps is compact, so it represents a (virtual) fundamental class that defines local GW invariants of the spin curve $(D, N)$. These local GW invariants depend only on the genus $h$ and the parity $p \equiv h^{0}(N)(\bmod 2)$, and GW invariants of Kähler surfaces with $p_{g}>0$ are the sum of local GW invariants associated to spin curves [LP1].

GW invariants count maps from connected domains, while Gromov-Taubes invariants count maps from not necessarily connected domains. These GT invariants can be obtained from GW invariants. Maulik and Pandharipande MP gave fascinating conjectural formulas for (descendent) local GT invariants of spin curve

Received by the editors May 26, 2009 and, in revised form, January 7, 2010, September 28, 2010 and May 9, 2011.

2010 Mathematics Subject Classification. Primary 53D45; Secondary 14N35.

(C)2012 American Mathematical Society 
$(D, N)$ for low degrees $(d=1,2)$ :

$$
\begin{aligned}
& G T_{1}^{l o c, h, p}\left(\prod_{i=1}^{n} \tau_{k_{i}}\left(F^{*}\right)\right)=(-1)^{p} \prod_{i=1}^{n} \frac{k_{i} !}{\left(2 k_{i}+1\right) !}(-2)^{-k_{i}}, \\
& G T_{2}^{l o c, h, p}\left(\prod_{i=1}^{n} \tau_{k_{i}}\left(F^{*}\right)\right)=(-1)^{p} 2^{h+n-1} \prod_{i=1}^{n} \frac{k_{i} !}{\left(2 k_{i}+1\right) !}(-2)^{k_{i}}
\end{aligned}
$$

(see Section 2 for a definition of descendent local GT invariants). Kiem and Li KL1, KL2, KL3 have since proved these formulas using their algebro-geometric version of local invariants. Observing that the formulas (0.2) for a genus zero spin curve directly follow from Proposition 2 of $[\mathrm{FP}$, they showed the following reduction theorem:

- for low degrees $(d=1,2)$ local GT invariants of higher genus spin curves can be reduced to local GT invariants of genus zero spin curve.

Their proof uses a sum formula (Theorem 4.2 of [KL1]) for degeneration obtained by certain blow-up plus explicit calculation of the invariant $G T_{2}^{l o c, h, p}\left(\tau\left(F^{*}\right)\right)$.

The aim of this paper is twofold. First, we give a proof of the sum formula for degeneration by blow-up in the context of symplectic geometry - this sum formula is the same as Kiem and Li's sum formula except for the constraints of relative invariants (see Theorem A below). Second, we also prove new sum formulas for the degeneration of spin curves in the case of degree $d=2$ (see Theorem B below). Then, in the proof of the above reduction theorem (cf. Section 4 of [KL1]), we can replace the calculation of invariant $G T_{2}^{l o c, h, p}(\tau)$ by Theorem B (see Section 9).

The novelty of our approach is to use GW invariants of ruled surfaces. Unlike the algebro-geometric approach, our local $\mathrm{GW}$ invariants of $(D, N)$ are, in fact, local contributions to GW invariants of the ruled surface $\mathbb{P}_{h}=\mathbb{P}\left(N \oplus \varnothing_{D}\right)$ that count maps whose images are close to the zero section $D$ of $\mathbb{P}_{h}$. A small neighborhood $U$ of $D$ in $\mathbb{P}_{h}$ is isomorphic to some neighborhood of the zero section in the total space $N_{D}$ of $N$. Together with this isomorphism and some bump function, the tautological holomorphic 2-form on $N_{D}$ induces an almost complex structure $J_{\alpha}$ on $\mathbb{P}_{h}$ satisfying the image localization property, namely

$$
\overline{\mathcal{M}}_{g, n}\left(U, d S, J_{\alpha}\right)=\overline{\mathcal{M}}_{g, n}(D, d),
$$

where $S$ is the section class of $\mathbb{P}_{h}$, i.e. $S=[D]$. The moduli space of $J_{\alpha}$-holomorphic maps into $U$ thus represents a (virtual) fundamental class that gives the local $\mathrm{GW}$ invariants of $(D, N)$. This description of local $\mathrm{GW}$ invariants is well suited to easily adapt the arguments in IP1, IP2 to a version of sum formulas for local GW invariants. The relative local GW invariants are simply the local contributions to the relative GW invariants of $\mathbb{P}_{h}$ that count maps into $U$ relative to a fixed fiber of $\mathbb{P}_{h}$. In terms of those relative invariants, sum formulas for local invariants directly follow from the main argument of IP2 for some cases.

Our relative invariants are, however, not given by a (virtual) fundamental class of relative moduli space that is needed to define descendent classes. To get around this issue, we relate descendent invariants to relative invariants with $\phi_{i}$ classes that are the first Chern classes of the relative cotangent bundles over the space of stable curves (see Proposition 5.1). Then, we use those relative invariants to show the sum formula for degeneration by blow-up. 
Let $\mathbb{F}_{0}=\mathbb{P}^{1} \times E$ be a ruled surface over $E=\mathbb{P}^{1}$. Then there is a unique section of $\mathbb{F}_{0}$ that passes through a given point. This simple observation enables us to apply the main argument of [IP2] for the symplectic fiber sum $\mathbb{P}_{h}=\mathbb{P}_{h} \# \mathbb{F}_{0}$ to obtain a sum formula for degeneration by blow-up. For any partition $m=\left(m_{1}, \cdots, m_{\ell}\right)$, we set

$$
\ell(m)=\ell, \quad|m|=\prod m_{i}, \quad m !=|\operatorname{Aut}(m)|,
$$

where $\operatorname{Aut}(m)$ is the symmetric group permuting equal parts of $m$. In Section 4 , we show :

Theorem A. Let $d \neq 0$ and $n_{1}+n_{2}=n$. Then

$$
\begin{aligned}
& G T_{d}^{l o c, h, p}\left(\prod_{i=1}^{n} \tau_{k_{i}}\left(F^{*}\right)\right) \\
= & \frac{1}{(d !)^{2}} \sum_{m} \frac{|m|}{m !} G T_{\left(1^{d}\right), m}^{l o c, h, p}\left(\prod_{i=1}^{n_{1}} \phi_{i}^{k_{i}}\left(F^{*}\right)\right) \cdot G T_{m,\left(1^{d}\right)}^{\mathbb{F}_{0}}\left(\prod_{i=1}^{n_{2}} \phi_{i}^{k_{n_{1}+i}}\left(F^{*}\right)\right),
\end{aligned}
$$

where the sum is over all partitions $m$ of d (see Section 3 for definition and notation of relative invariants).

Let $\mathbb{F}_{1}=\mathbb{P}\left(\mathcal{O}(1) \oplus \mathcal{O}_{E}\right)$ be a ruled surface over $E$. Unlike the case of $\mathbb{F}_{0}$, the infinite section plus a fiber represents the section class represented by the zero section of $\mathbb{F}_{1}$. This causes the main difficulty to derive general sum formulas of local GW invariants for degeneration of spin curves from the symplectic fiber sums

$$
\mathbb{P}_{h}=\mathbb{P}_{h_{1}} \# V_{1} \# \mathbb{F}_{1} \# V_{V_{2}} \mathbb{P}_{h_{2}} \quad \text { and } \quad \mathbb{P}_{h}=\mathbb{P}_{h-1} \underset{V_{1} \sqcup V_{2}}{\#} \mathbb{F}_{1} .
$$

However, when degree $d=2$, simple limiting arguments (see Section 6 and Lemma 7.3) allow us to apply the same arguments as in the proof of Theorem A. In Sections 7 and 8 , we show :

\section{Theorem B.}

(a) If $h=h_{1}+h_{2}$ and $p \equiv p_{1}+p_{2}(\bmod 2)$, then we have

$G T_{(2)}^{l o c, h, p}=(-1)^{p_{1}} 2^{h_{1}} G T_{(2)}^{l o c, h_{2}, p_{2}}+(-1)^{p_{2}} 2^{h_{2}} G T_{(2)}^{l o c, h_{1}, p_{1}}-(-1)^{p} 2^{h} G T_{(2)}^{l o c, 0,+}$.

(b) If $h \geq 2$ or if $(h, p)=(1,+)$, then we have

$$
G T_{(2)}^{l o c, h, p}=4 G T_{(2)}^{l o c, h-1, p}-(-1)^{p} 2^{h} G T_{(2)}^{l o c, 0,+} .
$$

\section{Moduli SPACES}

This section introduces moduli spaces of curves and maps. For $2 g+n \geq 3$, let $\overline{\mathcal{U}}_{g, n} \rightarrow \overline{\mathcal{M}}_{g, n}$ be the universal curve over the Deligne-Mumford space. Lifting to the moduli space of Prym curves ([Lo], [ACV] $)$, one may assume that $\overline{\mathcal{M}}_{g, n}$ is a manifold and every connected $n$ marked nodal curve $C$ of (arithmetic) genus $g$ has a stabilization $s t(C) \in \overline{\mathcal{M}}_{g, n}$ that is isomorphic to a fiber of $\overline{\mathcal{U}}_{g, n}$. After fixing an embedding $\overline{\mathcal{U}}_{g, n} \hookrightarrow \mathbb{P}^{N}$, one can obtain a map

$$
\phi: C \rightarrow \operatorname{st}(C) \rightarrow \overline{\mathcal{U}}_{g, n} \rightarrow \mathbb{P}^{N}
$$


Let $(X, \omega)$ be a compact symplectic manifold with an $\omega$-tamed almost complex structure $J$. A $C^{1}$-map $f: C \rightarrow X$ is stable if the energy

$$
E(f, \phi)=\frac{1}{2} \int|d f|^{2}+|d \phi|^{2}
$$

is positive on each (irreducible) component of $C$. An (irreducible) component of $C$ is called a ghost component if the restriction $f$ to that component represents a trivial homology class. Let $\nu$ be a section of the bundle $\operatorname{Hom}\left(\pi_{1}^{*} T \mathbb{P}^{N}, \pi_{2}^{*} T X\right)$ over $\mathbb{P}^{N} \times X$ satisfying $J \circ \nu=-\nu \circ J_{\mathbb{P}^{N}}$. A stable map $f$ is $(J, \nu)$-holomorphic if

$$
\frac{1}{2}(d f+J d f j)=(f, \phi)^{*} \nu,
$$

where $j$ is the complex structure on $C$. Denote by $\overline{\mathcal{M}}_{g, n}(X, A)$ the moduli space of $(J, \nu)$-holomorphic maps from nodal curves of (arithmetic) genus $g$ with $n$ marked points that represent the class $A$ (we often omit $(J, \nu)$ in notation). We also denote by

$$
\overline{\mathcal{M}}_{\chi, n}^{*}(X, A)
$$

the moduli space of $(J, \nu)$-holomorphic maps $f$ from possibly disconnected domains of Euler characteristic $\chi$ with no degree zero connected components, namely the restriction of $f$ to each "connected component" of its domain represents a nontrivial homology class.

For a finite set $A$, let $|A|$ denote the number of elements of $A$.

Remark 1.1. A stable map $f$ in the moduli space $\overline{\mathcal{M}}_{\chi, n}^{*}(X, A)$ might have ghost components. Let $C=C_{1} \cup C_{2}$ be the domain of $f$ such that $C_{1}$ is a connected curve that is a union of some ghost components of $f$. Then, the stability of $f$ implies that $2 g\left(C_{1}\right)+\ell+n_{1} \geq 3$, where $\ell=\left|C_{1} \cap C_{2}\right|$ and $n_{1}$ is the number of marked points on $C_{1}$.

The discussion below will be frequently used in subsequent arguments. Let $\phi_{i}$ be the first Chern class of line bundle over $\overline{\mathcal{M}}_{g, n}$ whose fiber over $\left(C,\left\{x_{i}\right\}\right)$ is $T_{x_{i}}^{*} C$. For a subset $I$ of $\{1, \ldots, n\}$, let $\delta_{I}$ denote the Poincaré dual of the fundamental class of the boundary stratum of $\overline{\mathcal{M}}_{g, n}$ that consists of nodal curves $C_{1} \cup C_{2}$ where $C_{1}$ has genus zero, $C_{2}$ has genus $g$ and the marked points on $C_{1}$ are precisely those labeled by $I$.

Consider the forgetful map

$$
\pi_{k}: \overline{\mathcal{M}}_{g, n+k} \rightarrow \overline{\mathcal{M}}_{g, n}
$$

that forgets the last $k$ marked points. For $1 \leq i \leq n$, we have

$$
\pi_{k}^{*} \phi_{i}=\phi_{i}-\sum \delta_{\{i\} \cup I}
$$

where the sum is over all $I \subset\{n+1, \ldots, n+k\}$ with $I \neq \emptyset$ (cf. Lemma 3.1 of (AC). The standard gluing map

$$
\eta: \overline{\mathcal{M}}_{g_{1}, n_{1}+1} \times \overline{\mathcal{M}}_{g_{2}, n_{2}+1} \rightarrow \overline{\mathcal{M}}_{g_{1}+g_{2}, n_{1}+n_{2}}
$$

is obtained by identifying the last marked point of the first component with the first marked point on the second component. For our purpose, we extend this gluing map to the cases where $2 g_{2}+n_{2}<2$. Denote by $\overline{\mathcal{M}}_{g, n}$ the space of one point when $2 g+n<3$ and note that

$$
\overline{\mathcal{M}}_{g, n_{1}+1} \times \overline{\mathcal{M}}_{0,1} \cong \overline{\mathcal{M}}_{g, n_{1}} \quad \text { and } \quad \overline{\mathcal{M}}_{g, n_{1}+1} \times \overline{\mathcal{M}}_{0,2} \cong \overline{\mathcal{M}}_{g, n_{1}+1} \text {. }
$$


Let $\eta$ be the forgetful map that forgets the last marked point when $2 g_{1}+n_{1} \geq 3$ and $\left(g_{2}, n_{2}\right)=(0,0)$, and let $\eta$ be the identity map when $\left(g_{2}, n_{2}\right)=(0,1)$. The following fact then directly follows from (1.2) and Lemma 3.3 of [AC].

Lemma 1.2 ([AC]). For $1 \leq i \leq n_{1}$ and $I \subset\left\{1, \ldots, n_{1}\right\}$ with $2 \leq|I|<n_{1}$, we have

(a) if $2 g_{1}+n_{1} \geq 3$ and $\left(g_{2}, n_{2}\right)=(0,0)$, then

$$
\eta^{*} \phi_{i}=\left(\phi_{i}-\delta_{\left\{i, n_{1}+1\right\}}\right) \otimes 1 \quad \text { and } \quad \eta^{*} \delta_{I}=\left(\delta_{I}+\delta_{I \cup\left\{n_{1}+1\right\}}\right) \otimes 1 ;
$$

(b) if either $\left(g_{2}, n_{2}\right)=(0,1)$ or $2 g_{j}+n_{j} \geq 2$ for $j=1,2$, then

$$
\eta^{*} \phi_{i}=\phi_{i} \otimes 1 \quad \text { and } \quad \eta^{*} \delta_{I}=\delta_{I} \otimes 1 .
$$

Lastly, we denote the space of curves with finitely many connected components, Euler class $\chi$ and $n$ marked points by

$$
\widetilde{\mathcal{M}}_{\chi, n}
$$

(cf. page 57 of [IP1]). This space is a disjoint union of the products of the spaces $\overline{\mathcal{M}}_{g_{j}, n_{j}}$ with $\sum\left(2-2 g_{j}\right)=\chi$ and $\sum n_{j}=n$ (including the unstable cases $2 g_{j}+n_{j}<$ $3)$. One can thus define $\phi_{i}$ classes and the boundary classes $\delta_{I}$ of $\widetilde{\mathcal{M}}_{\chi, n}$ in an obvious way.

\section{LOCAL GT INVARIANTS}

In this section, we introduce local Gromov-Taubes invariants of spin curves and set up notation for them. We will follow the definitions and notation in [RT2, [LT] and [P1]. Let $\pi: N \rightarrow D$ be a theta characteristic on a smooth curve $D$ of genus $h$. The canonical bundle of the total space $N_{D}$ of $N$ is then isomorphic to $\pi^{*} N$, so the tautological section of $\pi^{*} N$ gives a holomorphic 2-form $\alpha$ on $N_{D}$ whose zero set is the zero section $D \subset N_{D}$ (cf. [LP1]). The projectivization

$$
\mathbb{P}_{h}=\mathbb{P}\left(N \oplus \mathcal{O}_{D}\right)
$$

is a ruled surface over $D$. For small $\epsilon>0$ fix an isomorphism (denoted by $\Psi$ ) from a neighborhood of the zero section of $\mathbb{P}_{h}$ to the $3 \epsilon$-neighborhood of $D$ in $N_{D}$ by taking the zero section of $\mathbb{P}_{h}$ to $D$. Choose a bump function $\beta$ that is 1 on the $\epsilon$-neighborhood of $D$ in $N_{D}$ and vanishes on the complement of $2 \epsilon$-neighborhood of $D$. The pull-back 2-form $\Psi^{*}(\beta \alpha)$ is then a well-defined 2-form on $\mathbb{P}_{h}$. Fix a fiber $V$ of the ruled surface $\mathbb{P}_{h}$, and for small $\delta>0$ choose a bump function $\beta_{V}$ that is 1 on the complement of the $2 \delta$-neighborhood of $V$ and vanishes on the $\delta$-neighborhood of $V$. The 2-form

$$
\alpha_{V}=\beta_{V} \Psi^{*}(\beta \alpha)
$$

then induces, by (0.1), an almost complex structure $J_{V}$ on $\mathbb{P}_{h}$. Let $U \subset \mathbb{P}_{h}$ be the preimage of the $\epsilon$-neighborhood of $D$ in $N_{D}$ under the isomorphism $\Psi$. We also denote by $D$ the zero section of $\mathbb{P}_{h}$ and let $S$ be the section class represented by the zero section $D$ of $\mathbb{P}_{h}$.

Lemma 2.1. Let $U$ and $J_{V}$ be as above. Then every $J_{V}$-holomorphic map from a connected domain into $\bar{U}$ that represents the class $d S$ with $d \neq 0$ is, in fact, holomorphic and its image lies entirely in D, i.e.

$$
\overline{\mathcal{M}}_{\chi, n}^{*}\left(\bar{U}, d S, J_{V}\right)=\overline{\mathcal{M}}_{\chi, n}^{*}(D, d) .
$$


Proof. This proof is similar to that of Lemma 3.2 in [LP1]. Use the same notation $\alpha$ for the real part of the holomorphic 2 -form $\alpha$ on $N_{D}$. Let $f:(C, j) \rightarrow \bar{U}$ be a $J_{V}$-holomorphic map from a connected curve $C$ with complex structure $j$ that represents the class $d S$ where $d \neq 0$. For each point $q \in C$, let $\left\{e_{1}, e_{2}=j e_{1}\right\}$ be an orthonormal basis of $T_{q} C$. Then

$$
|\bar{\partial} f|^{2}=f^{*}\left|\beta_{V} \Psi^{*}(\beta \alpha)\right|^{2}|\partial f|^{2}=f^{*}\left(\beta_{V} \Psi^{*}(\beta \alpha)\right)\left(e_{1}, e_{2}\right) \leq f^{*}\left(\Psi^{*} \alpha\right)\left(e_{1}, e_{2}\right),
$$

where the two equalities follow from Proposition 1.3 of $[\mathrm{L}]$ and the inequality follows from the facts (i) $\Psi^{*} \beta \equiv 1$ on $\bar{U}$ and (ii) $0 \leq \beta_{V} \leq 1$. Since $\alpha$ is a real part of holomorphic 2-form, integrating over the domain shows $f$ is indeed holomorphic and the image of $f$ lies in the zero set of $\beta_{V} \Psi^{*}(\beta \alpha)$ in $\bar{U}$. Since $f$ represents the class $d S$, the image of $f$ must lie in the zero set of $\Psi^{*}(\beta \alpha)$ in $\bar{U}$ which is the zero section $D$. This completes the proof.

Remark 2.2. Let $\alpha$ be a holomorphic 2-form on $U$ and $g$ be any function on $U$ that satisfies $0 \leq g \leq 1$. Then, the inequality in (2.2) shows that for $\widetilde{\alpha}=g \alpha$ every $J_{\widetilde{\alpha}}$-holomorphic map into $U$ representing the class $d S(d \neq 0)$ is holomorphic and has its image lying in the zero set of $\widetilde{\alpha}$.

Let $d \neq 0$ and fix $(J, \nu)$ that is close to $\left(J_{V}, 0\right)$. Lemma 2.1 and the Gromov Compactness Theorem imply that the moduli space of $(J, \nu)$-holomorphic maps $\overline{\mathcal{M}}_{\chi, n}^{*}(U, d S)$ is compact. The construction of Li and Tian [LT] then defines the (virtual) fundamental class

$$
\left[\overline{\mathcal{M}}_{\chi, n}^{*}(U, d S)\right]^{v i r} \in H_{*}\left(\mathcal{M a p}_{\chi, n}\left(\mathbb{P}_{h}, d S\right) ; \mathbb{Q}\right)
$$

in the homology of the space $\mathcal{M} a p_{\chi, n}\left(\mathbb{P}_{h}, d S\right)$ of stable maps into $\mathbb{P}_{h}$ from nodal curves of Euler characteristic $\chi$ with $n$ marked points that represent the homology class $d S$.

Definition 2.3. The local (descendent) GT invariants of the spin curve $(D, N)$ of genus $h$ with parity $p \equiv h^{0}(N)(\bmod 2)$ are

$$
G T_{d}^{l o c, h, p}\left(\prod_{i=1}^{n} \tau_{k_{i}}\left(F^{*}\right)\right)=\left[\overline{\mathcal{M}}_{\chi, n}^{*}(U, d S)\right]^{v i r} \cap\left(\prod_{i=1}^{n} \psi_{i}^{k_{i}} \cup e v_{i}^{*}\left(F^{*}\right)\right),
$$

where $\psi_{i}$ is the Euler class of the bundle over $\mathcal{M a p}_{\chi, n}\left(\mathbb{P}_{h}, d S\right)$ whose fiber over $\left(f, C,\left\{x_{i}\right\}\right)$ is $T_{x_{i}}^{*} C, F^{*}$ is the Poincaré dual of the fiber class of $\mathbb{P}_{h}, e v_{i}$ is the evaluation map at the $i$-th marked point, and the Euler characteristic $\chi$ satisfies

$$
\sum k_{i}=d(1-h)-\frac{1}{2} \chi
$$

We will often write respectively + and - for $p \equiv 0(\bmod 2)$ and for $p \equiv 1(\bmod 2)$.

Remark 2.4. Let $\beta_{t}$ be a path from $\beta_{0}=\beta_{V}$ to $\beta_{1} \equiv 1$ with $0 \leq \beta_{t} \leq 1$ on $\mathbb{P}_{h}$ and let $J_{t}$ denote the almost complex structure induced from the 2 -form $\beta_{t} \Psi^{*}(\beta \alpha)$ by (0.1). The proof of Lemma 2.1 shows for $d \neq 0$ and for all $t$

$$
\overline{\mathcal{M}}_{\chi, n}^{*}\left(\bar{U}, d S, J_{t}\right)=\overline{\mathcal{M}}_{\chi, n}^{*}(D, d) .
$$

In particular, this shows $\overline{\mathcal{M}}_{\chi, n}^{*}\left(\bar{U}, d S, J_{t}\right)$ is compact for all $t$. It then follows from the standard cobordism argument (cf. Proposition 2.3 of [LT] that the (virtual) 
fundamental class (2.3) is independent of the choice of $J_{t}$. So, when $t=1$, the isomorphism $\Psi$ as above gives

$$
\begin{aligned}
{\left[\overline{\mathcal{M}}_{\chi, n}^{*}(U, d S)\right]^{v i r} \cap\left(\prod_{i=1}^{n} \psi_{i}^{k_{i}} \cup e v_{i}^{*}\left(F^{*}\right)\right) } & \\
= & {\left[\overline{\mathcal{M}}_{\chi, n}^{*}\left(N_{D}, d[D]\right)\right]^{v i r} \cap\left(\prod_{i=1}^{n} \psi_{i}^{k_{i}} \cup e v_{i}^{*}\left(\pi^{*} \gamma^{*}\right)\right) }
\end{aligned}
$$

where $\gamma^{*} \in H^{2}(D)$ is the Poincaré dual of the point class of $D$. Thus, given $\chi, n$ and $d \neq 0$ the invariant (2.4) depends only on the genus $h$ of $D$ and the parity $p=h^{0}(N)(\bmod 2)$.

The stabilization and evaluation at marked points defines a map

$$
\varepsilon=s t \times e v: \mathcal{M a p}_{\chi, n}\left(\mathbb{P}_{h}, d S\right) \rightarrow \widetilde{\mathcal{M}}_{\chi, n} \times\left(\mathbb{P}_{h}\right)^{n} .
$$

For the classes $\phi_{i}$ on $\widetilde{\mathcal{M}}_{\chi, n}$, we set

$$
G T_{d}^{l o c, h, p}\left(\prod_{i=1}^{n} \phi_{i}^{k_{i}}\left(F^{*}\right)\right)=\left[\overline{\mathcal{M}}_{\chi, n}^{*}(U, d S)\right]^{v i r} \cap\left(\prod_{i=1}^{n} s t^{*} \phi_{i}^{k_{i}} \cup e v_{i}^{*}\left(F^{*}\right)\right) .
$$

Now, suppose $(J, \nu)$ is generic (see page 10 of [RT2]). Then, the image of $\overline{\mathcal{M}}_{\chi, n}^{*}(U, d S)$ under the map (2.5) defines a homology class

$$
\left[\overline{\mathcal{M}}_{\chi, n}^{*}(U, d S)\right] \in H_{*}\left(\widetilde{\mathcal{M}}_{\chi, n} \times\left(\mathbb{P}_{h}\right)^{n} ; \mathbb{Q}\right)
$$

satisfying $\varepsilon_{*}\left[\overline{\mathcal{M}}_{\chi, n}^{*}(U, d S)\right]^{v i r}=\left[\overline{\mathcal{M}}_{\chi, n}^{*}(U, d S)\right]$ (cf. Remark 10.2 of [LP2]). So, we have

$$
G T_{d}^{l o c, h, p}\left(\prod_{i=1}^{n} \phi_{i}^{k_{i}}\left(F^{*}\right)\right)=\left[\overline{\mathcal{M}}_{\chi, n}^{*}(U, d S)\right] \cap \prod_{i=1}^{n} \phi_{i}^{k_{i}} \otimes\left(F^{*}\right)^{\otimes n} .
$$

Remark 2.5. If the spin curve $(D, N)$ has genus $h>0$, then there are no nonconstant holomorphic maps from genus zero curves to $D$. Hence, by the Gromov Compactness Theorem and Lemma 2.1, for every map $f$ in the moduli space $\overline{\mathcal{M}}_{\chi, n}^{*}(U, d S)$ every genus zero (irreducible) component is a ghost component. It thus follows from the stability and the relation between the $\psi_{i}$ class and the $s t^{*} \phi_{i}$ class (cf. KM] page 388) that

$$
G T_{d}^{l o c, h, p}\left(\prod_{i=1}^{n} \tau_{k_{i}}\left(F^{*}\right)\right)=G T_{d}^{l o c, h, p}\left(\prod_{i=1}^{n} \phi_{i}^{k_{i}}\left(F^{*}\right)\right) .
$$

We end this section with dimension zero local GT invariants for $d=1$ and 2 .

Lemma 2.6. $\quad G T_{1}^{l o c, h, p}=(-1)^{p} \quad$ and $\quad G T_{2}^{l o c, h, p}=(-1)^{p} 2^{h-1}$.

Proof. The dimension zero local GW invariants $G W_{d}^{l o c, h, p}$ and the dimension zero local GT invariants $G T_{d}^{l o c, h, p}$ are related as follows:

$$
1+\sum_{d>0} G T_{d}^{l o c, h, p} t^{d}=\exp \left(\sum_{d>0} G W_{d}^{l o c, h, p} t^{d}\right)
$$


(cf. Section 2 of [IP2]). The lemma thus follows from the fact

$$
G W_{1}^{l o c, h, p}=(-1)^{p} \quad \text { and } \quad G W_{2}^{l o c, h, p}=\frac{1}{2}\left[(-1)^{p} 2^{h}-1\right]
$$

(see Section 10 of [LP1]).

\section{RELATIVE LOCAL INVARIANTS}

In [IP1, GW invariants were generalized to relative GW invariants relative to the codimension two symplectic submanifold. Following IP1, we can define relative local invariants. A $(J, \nu)$-holomorphic map $f$ is called $V$-regular with a contact vector $s=\left(s_{1}, \ldots, s_{\ell}\right)$ if $f^{-1}(V)$ consists of the last $\ell$ ordered marked points $x_{n+1}, \ldots, x_{n+\ell}$ such that the image of $f$ has the contact order $s_{k}$ at $x_{n+k}$. Denote by

$$
\mathcal{M}_{\chi, n, s}^{V, *}(U, d S)
$$

the moduli space of $V$-regular $(J, \nu)$-holomorphic maps $f$ into $U$ with contact vector $s$ where the superscript $*$ also means $f$ has no degree zero connected components. For a contact vector $s=\left(s_{1}, \ldots, s_{\ell}\right)$, we write

$$
\operatorname{deg}(s)=\sum_{k=1}^{\ell} s_{k}, \quad \ell(s)=\ell, \quad|s|=\prod_{k=1}^{\ell} s_{k}
$$

and, noting there are no rim tori since $H_{1}(V)=0$ (cf. Remark 5.3 of [IP1]), we set

$$
V_{s}=\left\{\left(\left(v_{1}, s_{1}\right), \ldots,\left(v_{\ell}, s_{\ell}\right)\right) \mid v_{k} \in V\right\} .
$$

The moduli space of $V$-regular maps $\mathcal{M}_{\chi, n, s}^{V, *}(U, d S)$ also has an associated map

$$
\varepsilon_{s}=s t \times e v \times h_{s}: \mathcal{M}_{\chi, n}^{V, *}(U, d S) \rightarrow \widetilde{\mathcal{M}}_{\chi, n+\ell(s)} \times\left(\mathbb{P}_{h}\right)^{n} \times V_{s},
$$

where $e v$ is the evaluation map at the first $n$ marked points and $h_{s}$ is given by

$$
h_{s}\left(f, x_{1}, \ldots, x_{n+\ell}\right)=\left(\left(f\left(x_{n+1}\right), s_{1}\right), \ldots,\left(f\left(x_{n+\ell}\right), s_{\ell}\right)\right) .
$$

Observe that the (holomorphic) fiber $V$ of $\mathbb{P}_{h}$ is $J_{V}$-holomorphic since the 2-form $\alpha_{V}$ in (2.1) vanishes near $V$. The pair $\left(J_{V}, 0\right)$ is thus $V$-compatible in the sense of Definition 3.2 of [IP1. Now, choose a generic $V$-compatible $(J, \nu)$ that is sufficiently close to $\left(J_{V}, 0\right)$. Lemma 2.1, the Gromov Compactness Theorem and the relative GW theory of [IP1] then imply that the image of the moduli space $\mathcal{M}_{\chi, n, s}^{V, *}(U, d S)$ under the map (3.1) defines a homology class

$$
\left[\mathcal{M}_{\chi, n, s}^{V, *}(U, d S)\right] \in H_{*}\left(\widetilde{\mathcal{M}}_{\chi, n+\ell(s)} \times\left(\mathbb{P}_{h}\right)^{n} \times V_{s} ; \mathbb{Q}\right) .
$$

Let $\left\{\beta_{j}\right\}$ be a basis of $H_{*}(V)$. Then a basis of $H^{*}\left(V_{s}\right)$ is given by elements of the form

$$
C_{J, s}=C_{\beta_{j_{1}}, s_{1}} \otimes \cdots \otimes C_{\beta_{j_{\ell}}, s_{\ell}} .
$$

Lemma 2.1 and the Gromov Compactness Theorem imply that

$$
\left[\mathcal{M}_{\chi, n, s}^{V, *}(U, d S)\right] \cap C_{J, s}=0
$$

unless all $\beta_{j_{k}}$ are the fundamental class $[V]$ of $V$. Since $H_{1}(V)=0$, we can forget the ordering of the contact constraints $C_{J, s}$ by simply writing

$$
\prod_{j, b}\left(C_{\beta_{j}, b}\right)^{m_{j, b}}=C_{\beta_{j_{1}}, s_{1}} \cdots C_{\beta_{j_{\ell}}, s_{\ell}}
$$


with the relation $C_{\beta_{j}, b} \cdot C_{\beta_{i}, a}=C_{\beta_{i}, a} \cdot C_{\beta_{j}, b}$, where $m=\left(m_{j, b}\right)$ is a sequence of nonnegative integers determined by (3.5). If all $\beta_{j_{k}}=\beta_{j}$ for some $j$, then the sequence $m$ can be considered as a partition of the integer $d$. The (unordered) contact constraint (3.5) is then a pair of the Poincaré dual of $\beta_{j}$ and the partition $m$ of $d$, i.e. $m=\left(m_{1}, \ldots, m_{\ell}\right)$ with $m_{1} \leq m_{2} \leq \cdots \leq m_{\ell}$ and $\sum m_{j}=d$. Write $m=\left(1^{d}\right)$ if all $m_{j}=1$. In that case,

$$
|m|=\left|\left(1^{d}\right)\right|=1 \quad \text { and } \quad m !=\left(1^{d}\right) !=d ! .
$$

When all $\beta_{j_{k}}=\beta_{j}$ for some $j$, we write $C_{J, s}$ simply as $C_{\beta_{j}^{\ell}}$.

Remark 3.1. Given a partition $m=\left(m_{1}, \ldots, m_{\ell}\right)$ of $d$, there are $\ell ! / m !$ ordered sequences $s$ with $\tau(s)=m$ for some permutation $\tau$ in the symmetric group $S_{\ell}$.

Definition 3.2. For a partition $m$ of $d$ with $m=\tau(s)$ for some permutation $\tau$ in $S_{\ell(s)}$, we set

$$
G T_{m}^{l o c, h, p}\left(\prod_{i=1}^{n} \phi_{i}^{k_{i}}\left(F^{*}\right)\right)=\left[\mathcal{M}_{\chi, n, s}^{V, *}(U, d S)\right] \cap \prod_{i=1}^{n} \phi_{i}^{k_{i}} \otimes\left(F^{*}\right)^{n} \otimes C_{[V]^{\ell(m)}},
$$

where the Euler characteristic $\chi$ is given by

$$
\sum k_{i}=d(1-h)-\frac{1}{2} \chi+(\ell(m)-d) .
$$

Choose two distinct fibers $V_{1}$ and $V_{2}$ of $\mathbb{P}_{h}$ and let $V=V_{1} \sqcup V_{2}$. We can also define an almost complex structure $J_{V}$ that equals the Kähler structure of $\mathbb{P}_{h}$ near $V$ and satisfies Lemma 2.1. Thus, we can define relative local invariants

$$
G T_{m^{1}, m^{2}}^{l o c, h, p}\left(\prod_{i=1}^{n} \phi_{i}^{k_{i}}\left(F^{*}\right)\right)
$$

relative to $V$ (with contact vectors $m^{i}$ with $V_{i}$ ) for the class $d S$ with Euler characteristic $\chi$, where the Euler characteristic $\chi$ satisfies

$$
\sum k_{i}=d(1-h)-\frac{1}{2} \chi+\sum\left(\ell\left(m^{i}\right)-d\right) .
$$

Remark 3.3. The only genus zero spin curve is the even spin curve $\left(\mathbb{P}^{1}, \mathcal{O}(-1)\right)$. In this case, $S^{2}=-1$, and hence for $d \neq 0$ we have

$$
\overline{\mathcal{M}}_{\chi, n}^{*}\left(U, d S, J_{V}\right)=\overline{\mathcal{M}}_{\chi, n}^{*}\left(\mathbb{P}_{0}, d S, J_{V}\right) .
$$

This shows that degree $d$ local invariants of spin curve $\left(\mathbb{P}^{1}, \mathcal{O}(-1)\right)$ are the same as the GW invariants of $\mathbb{P}_{0}$ for the class $d S$. It also shows that relative local invariants are equal to the relative $\mathrm{GW}$ invariants of $\left(\mathbb{P}_{0}, V\right)$.

For simplicity, we set

$$
\overline{\mathcal{M}}=\overline{\mathcal{M}}_{\chi, n}^{*}(U, d S) \quad \text { and } \quad \mathcal{M}^{V}=\mathcal{M}_{\chi, n, m^{1}, m^{2}}^{V_{1}, V_{2}, *}(U, d S) .
$$

Noting the homology class (2.6) defines a map $H^{*}\left(\left(\mathbb{P}_{h}\right)^{n}\right) \rightarrow H_{*}\left(\widetilde{\mathcal{M}}_{\chi, n}\right)$, we set

$$
G T_{d, \chi}^{l o c, h, p}\left(\left(F^{*}\right)^{n}\right)=[\overline{\mathcal{M}}] \cap\left(F^{*}\right)^{\otimes n} \in H_{2 q}\left(\widetilde{\mathcal{M}}_{\chi, n}\right),
$$

where $q=d(1-h)-\frac{1}{2} \chi$. Similarly, we also set

$$
G T_{m^{1}, m^{2}, \chi}^{l o c, p}\left(\left(F^{*}\right)^{n}\right)=\left[\mathcal{M}^{V}\right] \cap\left(F^{*}\right)^{n} \bigotimes_{i=1}^{2} C_{\left[V_{i}\right]^{\ell\left(m^{i}\right)}} \in H_{2 r}\left(\widetilde{\mathcal{M}}_{\chi, n+\sum \ell\left(m^{i}\right)}\right),
$$

where $r=d(1-h)-\frac{1}{2} \chi+\sum\left(\ell\left(m^{i}\right)-d\right)$. 
Remark 3.4. Let $B$ be a geometric representative of the $n$ product of fiber classes $F^{\otimes n}$ of $\left(\mathbb{P}_{h}\right)^{n}$ in general position with respect to the evaluation map at marked points. Then the images of the cut-down moduli spaces $\overline{\mathcal{M}} \cap B$ and $\mathcal{M}^{V} \cap B$ under the stabilization map respectively define classes satisfying

$[\operatorname{st}(\overline{\mathcal{M}} \cap B)]=G T_{d, \chi}^{l o c, h, p}\left(\left(F^{*}\right)^{n}\right)$ and $\left[\operatorname{st}\left(\mathcal{M}^{V} \cap B\right)\right]=G T_{m^{1}, m^{2}, \chi}^{l o c h, p}\left(\left(F^{*}\right)^{n}\right)$.

For the ruled surface $\mathbb{F}_{0}=\mathbb{P}^{1} \times \mathbb{P}^{1}$, we use the same notation $F$ and $S$ for the fiber class and the section class, respectively. To save notation, we also use the same notation $V$ for the union of 2 distinct fibers $V_{1}$ and $V_{2}$ of the ruled surface $\mathbb{F}_{0}$. For partitions $m^{i}$ of $d$, denote by

$$
G T_{m^{1}, m^{2}}^{\mathbb{F}_{0}}\left(\prod_{i=1}^{n} \phi_{i}^{k_{i}}\left(F^{*}\right)\right)
$$

the relative GT invariants of $\left(\mathbb{F}_{0}, V\right)$ for the class $d S$ with Euler characteristic $\chi$ satisfying

$$
\sum k_{i}=d-\frac{1}{2} \chi+\left(\ell\left(m^{2}\right)-d\right),
$$

where the contact constraint with $V_{1}$ and $V_{2}$ are respectively $C_{p t^{\ell\left(m^{1}\right)}}$ and $C_{\left[V_{2}\right]^{\ell\left(m^{2}\right)}}$. It follows directly from Lemma 14.6 of [P2] that

$$
G T_{\left(1^{d}\right)}^{\mathbb{F}_{0}}=1 \quad \text { and } \quad G T_{\left(1^{d}\right),\left(1^{d}\right)}^{\mathbb{F}_{0}}=d !
$$

For the class $\left[\mathcal{M}_{\chi, n}^{V}\left(\mathbb{F}_{0}, d S\right)\right]$ that defines the relative invariants 3.7) of $\left(\mathbb{F}_{0}, V\right)$ we also set

$$
G T_{m^{1}, m^{2}, \chi}^{\mathbb{F}_{0}}\left(\left(F^{*}\right)^{n}\right)=\left[\mathcal{M}_{\chi, n}^{V}\left(\mathbb{F}_{0}, d S\right)\right] \cap\left(F^{*}\right)^{n} \otimes C_{p t^{\ell\left(m^{1}\right)}} \otimes C_{\left[V_{2}\right]^{\ell\left(m^{2}\right)}} .
$$

This is a homology class in $H_{2 t}\left(\widetilde{\mathcal{M}}_{\chi, n+\sum \ell\left(m^{i}\right)}\right)$, where $t=d-\frac{1}{2} \chi+\sum\left(\ell\left(m^{2}\right)-d\right)$.

\section{BlOW-UP AND SUM FORMUlA}

The aim of this section is to prove Theorem A in the Introduction. We will apply the limiting and smoothing arguments of IP2 to our local invariants. The proof consists of three steps.

Step 1. Fix a fiber $V_{0}$ of $\mathbb{P}_{h}$ and consider a degeneration

$$
\lambda: Z \stackrel{\sigma}{\longrightarrow} \mathbb{P}_{h} \times \mathbb{C} \longrightarrow \mathbb{C},
$$

where $\sigma: Z \rightarrow \mathbb{P}_{h} \times \mathbb{C}$ is the blow-up of $\mathbb{P}_{h} \times \mathbb{C}$ along $V_{0} \times\{0\}$ and the second map is projection onto the second factor. The central fiber $Z_{0}$ is the singular surface $\mathbb{P}_{h} \cup_{V_{0}} \mathbb{F}_{0}$, and general fibers $Z_{\lambda}(\lambda \neq 0)$ are isomorphic to $\mathbb{P}_{h}$ that is the symplectic fiber sum $\mathbb{P}_{h} \# V_{V_{0}} \mathbb{F}_{0}$. For $\lambda \neq 0$, let $D_{\lambda}$ denote the zero section of $Z_{\lambda}$, i.e., $D_{\lambda}=\sigma^{-1}(D \times\{\lambda\})$.

Choose a fiber $V_{1} \neq V_{0}$ of $\mathbb{P}_{h} \subset Z_{0}$ and a fiber $V_{2} \neq V_{0}$ of $\mathbb{F}_{0} \subset Z_{0}$ and set

$$
V=V_{1} \sqcup V_{0} \sqcup V_{2} \text {. }
$$

One can choose a (smooth) family $\tilde{V}_{\lambda}$ of disjoint union of two fibers of $Z_{\lambda} \simeq \mathbb{P}_{h}$ with $\tilde{V}_{0}=V_{1} \sqcup V_{2}$. Denote by $\mathcal{J}(Z)$ the space of all $(J, \nu)$ on $Z$ satisfying (i) each $Z_{\lambda}$ is $J$-invariant and (ii) the restriction of $(J, \nu)$ to $Z_{\lambda}(\lambda \neq 0)$ is $\tilde{V}_{\lambda}$-compatible and to $Z_{0}$ is $V$-compatible (cf. Lemma 2.3 of [IP2]). We will use the same notation $(J, \nu)$ for its restriction to each $Z_{\lambda}$. 
Fix a small $\delta>0$ and define a $\delta$-neck $Z(\delta)$ as a (normal) $\delta$-neighborhood of $V$ in $Z$. The energy of a map $f$ (more precisely of $(f, \phi)$ as in (1.1)) into $Z$ in the $\delta$-neck is

$$
E^{\delta}(f)=\frac{1}{2} \int|d f|^{2}+|d \phi|^{2},
$$

where the integral is over $f^{-1}(Z(\delta))$. By Lemma 1.5 of [IP1] there is a constant $c_{V}$ depending only on the restriction of $(J, \nu)$ to $V \subset Z$ such that every component of every $(J, \nu)$-holomorphic map into $V$ has energy greater than $c_{V}$. A $(J, \nu)$ holomorphic map $f$ into $Z$ is $\delta$-flat if the energy in the $\delta$-neck $E^{\delta}(f)$ is at most $c_{V} / 2$. Note that a $\delta$-flat map into $Z_{0}$ has no components mapped entirely into $V$.

Once and for all, fix $\chi, n, d \neq 0$, and for each $\lambda \neq 0$ we set

$$
\mathcal{M}\left(Z_{\lambda}\right)=\mathcal{M}_{\chi, n,\left(1^{d}\right),\left(1^{d}\right)}^{\tilde{V}_{\lambda}}\left(Z_{\lambda}, d S\right)
$$

Denote the set of $\delta$-flat maps in $\mathcal{M}\left(Z_{\lambda}\right)$ by $\mathcal{M}^{\delta}\left(Z_{\lambda}\right)$ and write

$$
\lim _{\lambda \rightarrow 0} \mathcal{M}^{\delta}\left(Z_{\lambda}\right)
$$

for the set of limits of sequences of $\delta$-flat maps in $\mathcal{M}^{\delta}\left(Z_{\lambda}\right)$ as $\lambda \rightarrow 0$. Since $\delta$-flatness is a closed condition, each map $f$ in the limit set (4.1) is also $\delta$-flat, and hence the domain of $f$ has no components mapped entirely into $V$. Consequently, we have

(a) $f$ splits as $f=\left(f_{1}, f_{2}\right)$, where $f_{1}$ and $f_{2}$ are respectively the $\left(V_{1} \sqcup V_{0}\right)$ regular map into $\mathbb{P}_{h}$ and the $\left(V_{0} \sqcup V_{2}\right)$-regular map into $\mathbb{F}_{0}$ and where each $f_{i}$ has contact vector $\left(1^{d}\right)$ with $V_{i}$ for $i=1,2$,

(b) $f^{-1}\left(V_{0}\right)$ consists of nodes $\left\{p_{1}, \ldots, p_{\ell}\right\}$ of the domain such that each $p_{i}$ has a well-defined multiplicity $s_{i}$ equal to the order of contact of the image of $f_{1}\left(\right.$ or $\left.f_{2}\right)$ with $V_{0}$ at $p_{i}$

(see Section 3 of [IP2]). Renumbering the nodes $\left\{p_{1}, \ldots, p_{\ell}\right\}$ gives $\ell$ ! ordered sequences $s=\left(s_{1}, s_{2}, \ldots, s_{\ell}\right)$. On the other hand, since for small $|\lambda|$ the $\delta$-flat maps in $\mathcal{M}^{\delta}\left(Z_{\lambda}\right)$ are $C^{0}$-close to $\delta$-flat maps in the limit set (4.1), to each map $f$ in $\mathcal{M}^{\delta}\left(Z_{\lambda}\right)$ one can assign ordered sequences $s$. Denote by $\mathcal{M}_{s}^{\delta}\left(Z_{\lambda}\right)$ the set of all such pair $(f, s)$ labeled by an ordered sequence $s$. Then, there are actions of symmetric groups $S_{\ell}$ such that

$$
\bigsqcup_{\ell}\left(\bigsqcup_{\ell(s)=\ell} \mathcal{M}_{s}^{\delta}\left(Z_{\lambda}\right)\right) / S_{\ell}=\mathcal{M}^{\delta}\left(Z_{\lambda}\right) .
$$

For $\mathbb{P}_{h}, \mathbb{F}_{0} \subset Z_{0}$ and each ordered sequence $s$ with $\operatorname{deg}(s)=d$, there is an evaluation map

$e v_{s}: \bigcup\left(\mathcal{M}_{\left.\chi_{1}, n_{1}, 1^{d}\right), s}^{V_{1}, V_{0}}\left(\mathbb{P}_{h}, d S-k F\right) \times \mathcal{M}_{\chi_{2}, n_{2}, s,\left(1^{d}\right)}^{V_{0}, V_{2}}\left(\mathbb{F}_{0}, d S+k F\right)\right) \longrightarrow V_{0}^{\ell(s)} \times V_{0}^{\ell(s)}$

that records the intersection points with the fiber $V_{0}$, where the union is over all $0 \leq k \leq h, n_{1}+n_{2}=n$ and $\chi=\chi_{1}+\chi_{2}-2 \ell(s)$. Let $\triangle_{s}$ be the diagonal of $V_{0}^{\ell(s)} \times V_{0}^{\ell(s)}$ and denote by

$$
\mathcal{K}_{s}^{\delta} \subset e v_{s}^{-1}\left(\triangle_{s}\right)
$$

the set of $\delta$-flat maps in $e v_{s}^{-1}\left(\triangle_{s}\right)$. Since each map in $e v_{s}^{-1}\left(\triangle_{s}\right)$ can be considered as a pair of a map $f$ into $Z_{0}=\mathbb{P}_{h} \cup_{V_{0}} \mathbb{F}_{0}$ satisfying $(a)$ and $(b)$ with an ordered sequence $s$, we have

$$
\lim _{\lambda \rightarrow 0} \bigsqcup_{s} \mathcal{M}_{s}^{\delta}\left(Z_{\lambda}\right) \subset \bigsqcup_{s} \mathcal{K}_{s}^{\delta} .
$$


Conversely, each map $f=\left(f_{1}, f_{2}\right) \in \mathcal{K}_{s}^{\delta}$ can be smoothed to produce maps in $\mathcal{M}_{s}^{\delta}\left(Z_{\lambda}\right)$ for small $|\lambda|$. Let $C_{1}$ and $C_{2}$ be the domains of $f_{1}$ and $f_{2}$ respectively. Identifying the $\ell(s)$ contact points with $V_{0}$ of $C_{1}$ with the $\ell(s)$ contact points with $V_{0}$ of $C_{2}$ determines a gluing map

$$
\widetilde{\mathcal{M}}_{\chi_{1}, n_{1}+\ell(s)+d} \times \widetilde{\mathcal{M}}_{\chi_{2}, n_{2}+\ell(s)+d} \longrightarrow \widetilde{\mathcal{M}}_{\chi, n+2 d},
$$

where $\chi_{i}=\chi\left(C_{i}\right)$ for $i=1,2$. For each $\ell(s)$, taking the union over all $\chi_{1}, \chi_{2}, n_{1}$ and $n_{2}$ defining a gluing map

$$
\xi_{\ell(s)}: \bigsqcup \widetilde{\mathcal{M}}_{\chi_{1}, n_{1}+\ell(s)+d} \times \widetilde{\mathcal{M}}_{\chi_{2}, n_{2}+\ell(s)+d} \longrightarrow \widetilde{\mathcal{M}}_{\chi, n+2 d},
$$

Theorem 10.1 of IP2 then gives:

Theorem 4.1 ([IP2]). For generic $(J, \nu) \in \mathcal{J}(Z)$ and for small $|\lambda|$, there is an $|s|$-fold covering

$$
\pi_{s, \lambda}: \mathcal{K}_{s}^{\delta}\left(Z_{\lambda}\right) \rightarrow \mathcal{K}_{s}^{\delta}
$$

with a commutative diagram (up to homotopy)

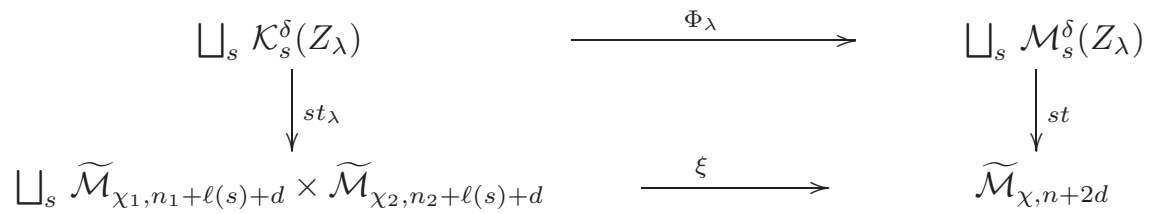

where the top arrow is an embedding, st $\lambda_{\lambda}=s t \circ \pi_{s, \lambda}$ and $\xi$ in the bottom arrow is given by the gluing maps $\xi_{\ell(s)}$. The construction of the smoothing map $\Phi_{\lambda}$ also shows that

$$
\lim _{\lambda \rightarrow 0} \Phi_{\lambda}\left(\tilde{f}_{\lambda}\right)=f, \quad \text { where } \quad \pi_{s, \lambda}\left(\tilde{f}_{\lambda}\right)=f .
$$

Step 2. Let $U$ be an open neighborhood of the zero section $D$ of $\mathbb{P}_{h}$ and $\alpha_{0}=\alpha_{V_{0}}$ be a 2 -form as in Section 2 (see the paragraph above Lemma 2.1). Regard the 2 -form $\alpha_{0}$ as a 2-form on $\mathbb{P}_{h} \times \mathbb{C}$ in an obvious way, and for small $\epsilon>0$ choose a bump function $\beta$ that is 1 on the complement of the $2 \epsilon$-neighborhood of $V_{1}$ in $Z$ and vanishes on an $\epsilon$-neighborhood of $V_{1}$ in $Z$. The 2 -form $\beta\left(p^{*} \alpha_{0}\right)$ then defines, again by the formula (0.1), an almost complex structure $J_{V}$ on $Z$. The pair $\left(J_{V}, 0\right) \in \mathcal{J}(Z)$ and the restriction of $J_{V}$ to $\mathbb{F}_{0}$ is the product complex structure of $\mathbb{F}_{0}$ since $p^{*} \alpha_{0}$ vanishes on some neighborhood of $\mathbb{F}_{0}$ in $Z$. Moreover, by Remark 2.2, we have

$$
\begin{aligned}
\overline{\mathcal{M}}_{\chi, n+2 d}^{*}\left(U_{\lambda}, d S, J_{V}\right) & =\overline{\mathcal{M}}_{\chi, n+2 d}^{*}\left(D_{\lambda}, d\right), \\
\overline{\mathcal{M}}_{\chi, n+2 d}^{*}\left(U_{0} \cap \mathbb{P}_{h}, d S, J_{V}\right) & =\overline{\mathcal{M}}_{\chi, n+2 d}^{*}(D, d),
\end{aligned}
$$

where $U_{\lambda}=p^{-1}(U \times\{\lambda\})$. Fix an open neighborhood $W$ of $D$ in $\mathbb{P}_{h}$ satisfying $\bar{W} \subset U$, and for each $\lambda$ set

$$
W_{\lambda}=p^{-1}(W \times\{\lambda\}) .
$$

The following fact is our key observation for the proof of Theorem A. 
Lemma 4.2. For $(J, \nu) \in \mathcal{J}(Z)$ sufficiently close to $\left(J_{V}, 0\right)$ and for small $|\lambda|>0$, we have

$$
\overline{\mathcal{M}}_{\chi, n+2 d}^{*}\left(U_{\lambda}, d S\right) \backslash \overline{\mathcal{M}}_{\chi, n+2 d}^{*}\left(W_{\lambda}, d S\right)=\emptyset .
$$

Proof. Suppose not. Then there exists a sequence of $\left(J_{k}, \nu_{k}\right)$-holomorphic maps $f_{k}$ into $U_{\lambda_{k}}$ with $\operatorname{Im}\left(f_{k}\right) \cap\left(U_{\lambda_{k}} \backslash W_{\lambda_{k}}\right) \neq \emptyset$ and with no degree zero connected components where $\lambda_{k}$ converges to 0 and $\left(J_{k}, \nu_{k}\right)$ converges to $\left(J_{V}, 0\right)$ as $k \rightarrow \infty$. The Gromov Compactness Theorem then implies that after passing to subsequences, $f_{k}$ converges to a $J_{V}$-holomorphic map $f$ into $Z_{0}$ such that (i) $\operatorname{Im}(f) \subset \bar{U}_{0}$ and (ii) $\operatorname{Im}(f) \cap\left(\bar{U}_{0} \backslash W_{0}\right) \neq \emptyset$. Since the limit map $f$ also has no degree zero connected components, (i) implies $f$ can be split as $f=\left(f_{1}, f_{2}\right)$, where $f_{1}$ and $f_{2}$ map into $\mathbb{P}_{h}$ and $\mathbb{F}_{0}$ respectively such that

$$
\operatorname{Im}\left(f_{1}\right) \cap V_{0}=\operatorname{Im}\left(f_{2}\right) \cap V_{0} .
$$

It follows from (4.7) that $\operatorname{Im}\left(f_{1}\right) \subset D$ and hence $\operatorname{Im}\left(f_{2}\right) \cap V_{0}=D \cap V_{0}$. Then, since the restriction of $J_{V}$ on $\mathbb{F}_{0}$ is the product complex structure, $\operatorname{Im}\left(f_{2}\right)$ lies in the section of $\mathbb{F}_{0}$ passing through the intersection point $D \cap V_{0}$. We have $\operatorname{Im}(f) \subset W_{0}$, which contradicts (ii).

Fix $(J, \nu) \in \mathcal{J}(Z)$ sufficiently close to $\left(J_{V}, 0\right)$, and for small $|\lambda|$ set

$$
\mathcal{M}_{s}^{\delta, *}\left(U_{\lambda}\right)=\left\{(f, s) \in \mathcal{M}_{s}^{\delta}\left(Z_{\lambda}\right) \mid f \in \mathcal{M}_{\chi, n,\left(1^{d}\right),\left(1^{d}\right)}^{\tilde{V}_{\lambda}, *}\left(U_{\lambda}, d S\right)\right\} .
$$

Consider the restriction of the evaluation map (4.3) :

$e v_{s, U_{0}}^{*}: \bigcup\left(\mathcal{M}_{\chi_{1}, n_{1},\left(1^{d}\right), s}^{V_{1}, V_{0}, *}\left(\mathbb{P}_{h} \cap U_{0}, d S\right) \times \mathcal{M}_{\chi_{2}, n_{2}, s,\left(1^{d}\right)}^{V_{0}, V_{1}, *}\left(\mathbb{F}_{0} \cap U_{0}, d S\right)\right) \longrightarrow V_{0}^{\ell(s)} \times V_{0}^{\ell(s)}$,

where the union is over all $n_{1}+n_{2}=n$ and $\chi=\chi_{1}+\chi_{2}-2 \ell(s)$.

Remark 4.3. Let $q$ be the intersection point of $D$ and $V$ in $Z_{0}=\mathbb{P}_{h} \cup_{V_{0}} \mathbb{F}_{0}$. Then there is a unique section $E_{q}$ of $\mathbb{F}_{0}$ that lies in $\mathbb{F}_{0} \cap U_{0}$ and intersects with $V_{0}$ at the point $q$. Choose $\ell(s)$ points $\left\{q_{j}\right\}$ in $V_{0} \cap\left(\mathbb{F}_{0} \cap U_{0}\right)$ that are sufficiently close to $q$. Denote by

$$
\mathcal{M}_{\chi_{2}, n_{2}, s,\left(1^{d}\right)}^{V_{0}, V_{2}, *}\left(\mathbb{F}_{0}, d S\right) \cap\left\{q_{j}\right\}
$$

the cut-down moduli space of $\left(V_{0} \sqcup V_{2}\right)$-regular $(J, \nu)$-holomorphic maps $f$ with $\ell(s)$ contact points $\left\{x_{n+j}\right\}$ (with $V_{0}$ ) satisfying $f\left(x_{n+j}\right)=q_{j}$. Since every holomorphic map representing the class $d S$ and passing through the point $q$ has its image in $E_{q}$, by the Gromov Compactness Theorem, we have

$$
\mathcal{M}_{\chi_{2}, n_{2}, s,\left(1^{d}\right)}^{V_{0}, V_{2}, *}\left(\mathbb{F}_{0} \cap U_{0}, d S\right) \cap\left\{q_{j}\right\}=\mathcal{M}_{\left.\chi_{2}, n_{2}, s, 1^{d}\right)}^{V_{0}, V_{2}, *}\left(\mathbb{F}_{0}, d S\right) \cap\left\{q_{j}\right\} .
$$

This shows that local invariants of $\mathbb{F}_{0}$ counting maps into $U_{0}$ with point constraints equal the standard invariants of $\mathbb{F}_{0}$ with points constraints.

Let $\mathcal{K}_{s, U_{0}}^{\delta, *}=\mathcal{K}_{s}^{\delta} \cap\left(e v_{s, U_{0}}^{*}\right)^{-1}\left(\triangle_{s}\right)$. Lemma 4.2 and (4.4) imply

$$
\begin{gathered}
\lim _{\lambda \rightarrow 0} \bigsqcup_{s} \mathcal{M}_{s}^{\delta, *}\left(U_{\lambda}\right) \subset \bigsqcup_{s} \mathcal{K}_{s, U_{0}}^{\delta, *}, \\
\lim _{\lambda \rightarrow 0} \bigsqcup_{s}\left(\mathcal{M}_{s}^{\delta}\left(Z_{\lambda}\right) \backslash \mathcal{M}_{s}^{\delta, *}\left(U_{\lambda}\right)\right) \cap \bigsqcup_{s} \mathcal{K}_{s, U_{0}}^{\delta, *}=\emptyset .
\end{gathered}
$$


Consequently, by (4.5), (4.6) and (4.9), for the restriction $\Phi_{\lambda}^{\text {loc }}$ of the smoothing $\operatorname{map} \Phi_{\lambda}$ to

$$
\mathcal{K}_{s, U_{0}}^{\delta, *}\left(U_{\lambda}\right)=\pi_{s, \lambda}^{-1}\left(\mathcal{K}_{s, U_{0}}^{\delta, *}\right),
$$

we have a commutative diagram (up to homotopy):

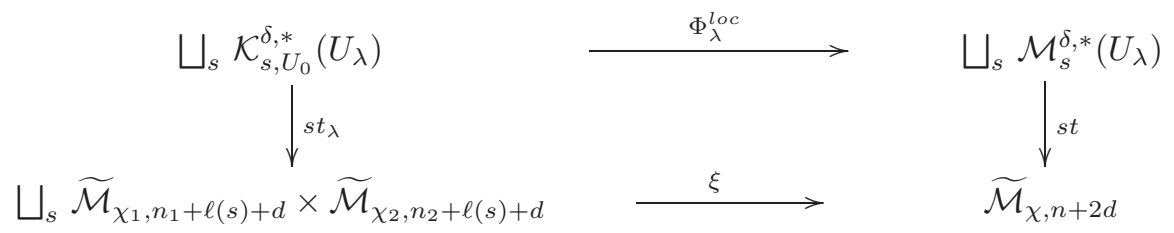

Step 3. The commutative diagram (4.10) leads to a sum formula for local invariants for the sum $\left(\mathbb{P}_{h}, V_{1} \sqcup V_{2}\right)$ of $\left(\mathbb{P}_{h}, V_{1} \sqcup V_{0}\right)$ and $\left(\mathbb{F}_{0}, V_{0} \sqcup V_{2}\right)$ along $V_{0}$. We first assume that all maps in

$$
\mathcal{M}_{\chi, n,\left(1^{d}\right),\left(1^{d}\right)}^{\tilde{V}_{\lambda}, *}\left(U_{\lambda}, d S\right)
$$

are $\delta$-flat when $|\lambda|$ is small. For fixed $n_{1}+n_{2}=n$, one can choose a continuous family of geometric representatives $B_{\lambda}$ disjoint with $\tilde{V}_{\lambda}$ satisfying:

- each $B_{\lambda}(\lambda \neq 0)$ is a geometric representative of the $n$ product of fiber classes $F^{\otimes n}=F \otimes \cdots \otimes F$ of $\left(Z_{\lambda}\right)^{n} \simeq\left(\mathbb{P}_{h}\right)^{n}$,

- $B_{0}=B_{\mathbb{P}_{h}} \sqcup B_{\mathbb{F}_{0}}$, where $B_{\mathbb{P}_{h}}$ and $B_{\mathbb{F}_{0}}$ are geometric representatives of the classes $F^{\otimes n_{1}}$ of $\left(\mathbb{P}_{h}\right)^{n_{1}}$ and $F^{\otimes n_{2}}$ of $\left(\mathbb{F}_{0}\right)^{n_{2}}$ respectively.

It now follows from the diagram (4.10) that

$$
\begin{aligned}
& {\left[\operatorname{st}\left(\mathcal{M}_{\chi, n,\left(1^{d}\right),\left(1^{d}\right)}^{\tilde{V}_{\lambda}, *}\left(U_{\lambda}, d S\right) \cap B_{\lambda}\right)\right] } \\
= & \sum_{s} \frac{|s|}{\ell(s) !}\left(\xi_{\ell(s)}\right)_{*}\left[s t\left(\left(e v_{s, U_{0}}^{*}\right)^{-1}\left(\triangle_{s}\right) \cap B_{0}\right)\right] \in H_{*}\left(\widetilde{\mathcal{M}}_{\chi, n+2 d}\right),
\end{aligned}
$$

where the sum is over all $\ell(s)=d$. Here, the factor $|s|$ is the degree of the covering map $\pi_{s, \lambda}$, the factor $\ell(s)$ ! reflects the fact that each map in the space $\mathcal{M}_{s}^{\delta, *}\left(U_{\lambda}\right)$ in the diagram (4.10) is a labeled map as in (4.2) and the classes $[s t(\cdot)]$ are defined by the images of cut-down moduli spaces under the stabilization map as in Remark 3.4

In general, if there are maps in the space (4.11) that are not $\delta$-flat, then there is a correction term in (4.12) given by three $S$-matrices in $\mathbb{F}_{0}$ (cf. Definition 11.3 of [IP2]) for $V_{1}, V_{0}$ and $V_{2}$. In our case, since the constraint $\left(F^{*}\right)^{n}$ is supported off the neck, Lemma 14.6 of [P2] and Theorem 12.3 of [P2] imply that the correction term is trivial. Consequently, by the splitting of the diagonal $\triangle_{s}$, together with (3.4), (4.8) and Remark 3.1 it follows from (4.12) that

$$
\begin{aligned}
& G T_{\left(1^{d}\right),\left(^{d}\right)}^{l o c, h, p}\left(\prod_{i=1}^{n} \phi_{i}^{k_{i}}\left(F^{*}\right)\right)=G T_{\left(1^{d}\right),\left(1^{d}\right), \chi}^{l o c, h, p}\left(\left(F^{*}\right)^{n}\right) \cap \prod_{i=1}^{n} \phi_{i}^{k_{i}}\left(F^{*}\right) \\
= & \sum \frac{|m|}{m !} G T_{\left(1^{d}\right), m, \chi_{1}}^{l o c, p}\left(\left(F^{*}\right)^{n_{1}}\right) \otimes G T_{m,\left(1^{d}\right), \chi_{2}}^{\mathbb{F}_{0}}\left(\left(F^{*}\right)^{n_{2}}\right) \cap\left(\xi_{\ell(m)}\right)^{*}\left(\prod_{i=1}^{n} \phi_{i}^{k_{i}}\right),
\end{aligned}
$$

where the sum is over all partitions $m$ of $d$ and $\chi_{1}+\chi_{2}-2 \ell(m)=\chi$ (cf. Theorem 12.3 of [P2]). 
Proof of Theorem A. Let $f=\left(f_{1}, f_{2}\right)$ be a map that contributes to the right-hand side of (4.13). Then we have

- every connected component of the domain of the $\left(V_{0} \sqcup V_{i}\right)$-regular map $f_{i}$ has at least two contact (marked) points with $V_{0} \sqcup V_{i}$,

- every connected component of the domain of $f_{2}$ has exactly one contact marked point with $V_{0}$; the contact constraint with $V_{0}$ is $C_{p t^{\ell(m)}}$ (see (3.7)) and the image of each connected component can't pass through more than two distinct points on $V_{0}$.

Noting that the gluing map $\xi_{\ell(m)}$ is the map obtained by successively applying gluing maps as in (1.3) to connected components, by Lemma 1.2(b) we have

$$
\left(\xi_{\ell(m)}\right)^{*}\left(\prod_{i=1}^{n} \phi_{i}^{k_{i}}\right)=\prod_{i=1}^{n_{1}} \phi_{i}^{k_{i}} \otimes \prod_{i=1}^{n_{2}} \phi_{i}^{k_{n_{1}+i}}
$$

The sum formula (4.13) together with (4.14) gives

$$
\begin{aligned}
G T_{\left(1^{d}\right),\left(1^{d}\right)}^{l o c, h, p}\left(\prod_{i=1}^{n} \phi_{i}^{k_{i}}\left(F^{*}\right)\right)= & \sum_{m} \frac{|m|}{m !} G T_{\left(1^{d}\right), m}^{l o c, h, p}\left(\prod_{i=1}^{n_{1}} \phi_{i}^{k_{i}}\left(F^{*}\right)\right) \\
& \cdot G T_{m,\left(1^{d}\right)}^{\mathbb{F}_{0}}\left(\prod_{i=1}^{n_{2}} \phi_{i}^{k_{n_{1}+i}}\left(F^{*}\right)\right) .
\end{aligned}
$$

Now, Theorem A follows from (4.15) and Proposition 5.1] in the next section.

\section{Descendent inVariants VS. RElative invariants}

The aim of this section is to show:

Proposition 5.1. $G T_{d}^{l o c, h, p}\left(\prod_{i=1}^{n} \tau_{k_{i}}\left(F^{*}\right)\right)=\frac{1}{(d !)^{2}} G T_{\left(1^{d}\right),\left(1^{d}\right)}^{l o c, h, p}\left(\prod_{i=1}^{n} \phi_{i}^{k_{i}}\left(F^{*}\right)\right)$.

By the relation of GT and GW invariants (cf. Section 2 of [IP2]), it suffices to prove Proposition 5.1 for local GW invariants that count maps with connected domains. Let $G W_{d}^{l o c, h, p}(\cdot)$ and $G W_{m^{1}, m^{2}}^{l o c, h,}(\cdot)$ denote absolute and relative local GW invariants and let

$$
\pi=\pi_{2 d}: \overline{\mathcal{M}}_{g, n+2 d} \rightarrow \overline{\mathcal{M}}_{g, n}
$$

be the forgetful map that forgets the last $2 d$ marked points.

Lemma 5.2. If $\sum k_{i}=0$ or $n \geq 3$, then we have

$$
G W_{d}^{l o c, h, p}\left(\prod_{i=1}^{n} \phi_{i}^{k_{i}}\left(F^{*}\right)\right)=\frac{1}{(d !)^{2}} G W_{\left(1^{d}\right),\left(1^{d}\right)}^{l o c, p}\left(\prod_{i=1}^{n} \pi^{*} \phi_{i}^{k_{i}}\left(F^{*}\right)\right) .
$$

Proof. Consider the symplectic fiber sum $\mathbb{P}_{h}=\mathbb{F}_{0} \#_{V_{1}} \mathbb{P}_{h} \#_{V_{2}} \mathbb{F}_{0}$, where $V_{1}$ and $V_{2}$ are two distinct fibers of $\mathbb{P}_{h}$. This sum can be obtained by blowing up $\mathbb{P}_{h} \times \mathbb{C}$ along $\left(V_{1} \cup V_{2}\right) \times\{0\}$. The same arguments of Section 4 thus give a sum formula that is 
analogous to 4.13):

$$
\begin{gathered}
G T_{d}^{l o c, h, p}\left(\prod_{i=1}^{n} \phi_{i}^{k_{i}}\left(F^{*}\right)\right) \\
=\sum \frac{\left|m^{1}\right|\left|m^{2}\right|}{m^{1 !} ! m^{2} !} G T_{m^{1}, \chi_{1}}^{\mathbb{F}_{0}} \otimes G T_{m^{1}, m^{2}, \chi_{0}}^{l o c, h, p}\left(\left(F^{*}\right)^{n}\right) \\
\otimes G T_{m^{2}, \chi_{2}}^{\mathbb{F}_{0}} \cap\left(\xi_{\ell\left(m^{1}\right), \ell\left(m^{2}\right)}\right)^{*}\left(\prod_{i=1}^{n} \phi_{i}^{k_{i}}\right),
\end{gathered}
$$

where the sum is over all $\chi_{1}+\chi_{0}+\chi_{2}-2 \ell\left(m^{1}\right)-2 \ell\left(m^{2}\right)=2 d(1-h)-2 \sum k_{i}$ and $\xi_{\ell\left(m^{1}\right), \ell\left(m^{2}\right)}$ is the gluing map obtained by identifying contact points of domains (see above Theorem 4.1). If $\sum k_{i}=0$, then for $k=1,2$, by dimension count, we have

$$
0=2 d-\frac{1}{2} \chi_{k}+\left(\ell\left(m^{k}\right)-d\right)-\ell\left(m^{k}\right)=d-\frac{1}{2} \chi_{k} .
$$

This shows $\chi_{k}=2 d$, and hence $m^{k}=\left(1^{d}\right)$. Thus, (5.2) for $n=0$ follows from (3.8) and (5.3).

Assume $n \geq 3$ and let $f=\left(f_{1}, f_{0}, f_{2}\right)$ be a map that contributes to the righthand side of (5.3). In order to obtain a sum formula for local GW invariants, we assume that the domain of $f$ is connected. We have

- since all marked points of $f$ map into the middle $\mathbb{P}_{h}$ side, the domain of $f_{k}$ $(k=1,2)$ mapped into $\mathbb{F}_{0}$ has no marked points except contact points,

- as in the proof of Theorem A, every connected component of the domain of $f_{k}$ has one contact point with $V_{k}$.

It follows that the domain of $f_{0}$ is connected and the gluing map $\xi=\xi_{\ell\left(m^{1}\right), \ell\left(m^{2}\right)}$ can be obtained by composing gluing maps as in (1.3) with $n_{2}=0$ :

$$
\eta: \overline{\mathcal{M}}_{g_{1}, n_{1}+1} \times \overline{\mathcal{M}}_{g_{2}, 1} \rightarrow \overline{\mathcal{M}}_{g_{1}+g_{2}, n_{1}+1},
$$

where $n_{1} \geq n \geq 3$. By Lemma 1.2(a) for $g_{2}=0$ and by Lemma 1.2(b) for $g_{2} \geq 1$, one can see that the pull-back class $\xi^{*} \phi_{i}$ restricts to the trivial class on two $\mathbb{F}_{0}$ sides and hence $f_{k}$ is constrained by only $\ell\left(m^{k}\right)$ point contact constraints. The dimension count (5.4) then shows $\chi_{k}=2 d$ and $m^{k}=\left(1^{d}\right)$, and hence $\xi$ is a composition of gluing maps $\eta$ as above with $\left(g_{2}, n_{2}\right)=(0,0)$. Consequently, again by Lemma 1.2(a), we have

$$
\xi^{*} \phi_{i}=1 \otimes\left(\phi_{i}-\sum \delta_{\{i\} \cup I}\right) \otimes 1,
$$

where the sum is over all $I \subset\{n+1, \ldots, n+2 d\}$ with $I \neq \emptyset$. On the other hand, (1.2) shows

$$
\pi^{*} \phi_{i}=\phi_{i}-\sum \delta_{\{i\} \cup I} .
$$

Now, (5.2) for $n \geq 3$ follows from (3.8), (5.3), (5.5) and (5.6).

Remark 5.3. The same argument of the proof of Lemma 5.2 applies to various sum formulas for the fiber sum of $\mathbb{P}_{h}$ and $\mathbb{F}_{0}$. In particular, for dimension zero local invariants (i.e. $\sum k_{i}=0$ ), one can use (3.8) and the dimension count (5.4) to show

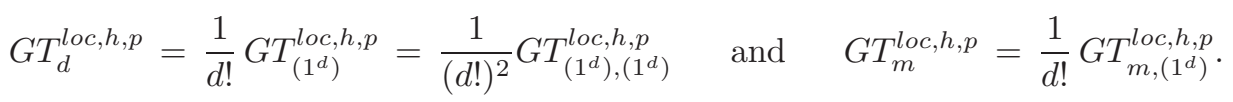


Lemma 5.4. If $\sum k_{i}=0$ or $n \geq 3$, then we have

$$
G W_{d}^{l o c, h, p}\left(\prod_{i=1}^{n} \tau_{k_{i}}\left(F^{*}\right)\right)=\frac{1}{(d !)^{2}} G W_{\left(1^{d}\right),\left(1^{d}\right)}^{l o c, h, p}\left(\prod_{i=1}^{n} \phi_{i}^{k_{i}}\left(F^{*}\right)\right) .
$$

Proof. If $\sum k_{i}=0$, then (5.7) follows from Lemma 5.2. Assume $n \geq 3$ and $h>0$ (we will give a proof in the appendix for the case when $h=0$ ). Let $V=V_{1} \cup V_{2}$ be a union of two distinct fibers of $\mathbb{P}_{h}$ and denote by

$$
\mathcal{M}^{V}=\mathcal{M}_{g, n,\left(1^{d}\right),\left(1^{d}\right)}^{V}(U, d S)
$$

the local relative GW moduli space. Let $B$ be a product of $n$ generic fibers of $\mathbb{P}_{h}$, each of which is disjoint with $V$, and let $f$ be a limit map of a sequence in the cutdown moduli space $C \mathcal{M}^{V} \cap B$ where $C \mathcal{M}^{V}$ is the closure of $\mathcal{M}^{V}$ in $\overline{\mathcal{M}}_{g, n+2 d}(U, d S)$. Then, Remark 2.5 shows that every genus zero irreducible component of $f$ maps entirely into either $B$ or $V$. This implies that for $1 \leq i \leq n$ and for any $I \subset$ $\{n+1, \ldots, n+2 d\}$ with $I \neq \emptyset$ we have

$$
\left[\mathcal{M}^{V}\right] \cap \delta_{\{i\} \cup I} \otimes\left(F^{*}\right)^{n}=0 .
$$

Therefore, (5.7) for $h>0$ follows from (2.7), Lemma 5.2, (5.6) and and (5.8).

Proof of Proposition 5.1. We will show that (5.7) holds for all $n$. By (4.15), we have

$$
\begin{aligned}
G W_{\left(1^{d}\right),\left(11^{d}\right)}^{l o c, h, p}\left(\prod_{i=1}^{n} \phi_{i}^{k_{i}}\left(F^{*}\right) F^{*} F^{*}\right) & =\frac{1}{d !} G W_{\left(1^{d}\right),\left(1^{d}\right)}^{l o c, h, p}\left(\prod_{i=1}^{n} \phi_{i}^{k_{i}}\left(F^{*}\right)\right) \cdot G T_{\left(1^{d}\right),\left(1^{d}\right)}^{\mathbb{F}_{0}}\left(F^{*} F^{*}\right) \\
& =d^{2} G W_{\left(1^{d}\right),\left(1^{d}\right)}^{l o c, h, p}\left(\prod_{i=1}^{n} \phi_{i}^{k_{i}}\left(F^{*}\right)\right),
\end{aligned}
$$

where the second equality follows from Divisor Axiom and (3.8). On the other hand, one can see that the generalized Divisor Axiom (cf. Lemma 1.4 of [KM] for descendant GW invariants also holds for descendant local invariants. Thus, we have

$$
\begin{aligned}
& G W_{d}^{l o c, h, p}\left(\prod_{i=1}^{n} \tau_{k_{i}}\left(F^{*}\right)\right)=\frac{1}{d^{2}} G W_{d}^{l o c, h, p}\left(\prod_{i=1}^{n} \tau_{k_{i}}\left(F^{*}\right) F^{*} F^{*}\right) \\
= & \frac{1}{d^{2}(d !)^{2}} G W_{\left(1^{d}\right),\left(1^{d}\right)}^{l o c, h, p}\left(\prod_{i=1}^{n} \phi_{i}^{k_{i}}\left(F^{*}\right) F^{*} F^{*}\right)=\frac{1}{(d !)^{2}} G W_{\left(1^{d}\right),\left(1^{d}\right)}^{l o c, h, p}\left(\prod_{i=1}^{n} \phi_{i}^{k_{i}}\left(F^{*}\right)\right),
\end{aligned}
$$

where the second equality follows from Lemma 5.4 and the last from (5.9). This completes the proof.

\section{Local CONTRIBUtions to GT inVARIANTS OF RULED SURFACES}

Let $\pi: \mathbb{F}_{1}=\mathbb{P}\left(\mathcal{O}_{E}(1) \oplus \mathcal{O}_{E}\right) \rightarrow E$ be a ruled surface over $E=\mathbb{P}^{1}$. This section describes local contributions to GW invariants of $\mathbb{F}_{1}$ that are needed for the proof of Theorem B. We also denote by $E$ the zero section of $\mathbb{F}_{1}$ and by $S$ the section class represented by the zero section $E$. 
Remark 6.1. Let $f: C \rightarrow \mathbb{F}_{1}$ be a holomorphic map from a smooth domain $C$ that represents the class $d S$. Then $f$ defines a holomorphic section $\xi$ of the line bundle $(\pi \circ f)^{*} \mathcal{O}_{E}(1)$ over $C$. The zero set $Z(\xi)$ of $\xi$ is the preimage $f^{-1}(E)$ of the zero section $E$, so if the image of $f$ does not lie in $E$ (i.e. $\xi \neq 0$ ), then

$$
\left|f^{-1}(E)\right|=|Z(\xi)| \leq \# Z(\xi)=\operatorname{deg}\left((\pi \circ f)^{*} \mathcal{O}_{E}(1)\right)=d,
$$

where $\# Z(\xi)$ is the number of zeros of $\xi$ counted with multiplicities.

Choose distinct fibers $V_{1}, V_{0}$ and $V_{2}$ of $\mathbb{F}_{1}$ and set

$$
V=V_{1} \sqcup V_{0} \sqcup V_{2} \text {. }
$$

Remark 6.2. Let $f: \mathbb{P}^{1} \rightarrow \mathbb{F}_{1}$ be a holomorphic map that represents the class $2 S$. If $f$ has a contact vector (2) with $V_{i}$ at $p_{i} \in \mathbb{P}^{1}$, then for the composition map

$$
\mathbb{P}^{1} \stackrel{f}{\longrightarrow} \mathbb{F}_{1} \stackrel{\pi}{\longrightarrow} E=\mathbb{P}^{1}
$$

the point $p_{i}$ is a ramification point of multiplicity two. Thus $f^{-1}(V)$ consists of at least four points. Otherwise, $\pi \circ f$ is a holomorphic map of degree 2 with three ramification points, which is impossible by the Riemann-Hurwitz formula.

Remark 6.1 and Remark 6.2 immediately give :

Lemma 6.3. Let $f$ be a holomorphic map into $\mathbb{F}_{1}$ from a smooth domain of genus $g$ representing the class $d S$ and satisfying

$$
\operatorname{Im}(f) \cap\left(V_{1} \sqcup V_{2}\right)=\left(V_{1} \sqcup V_{2}\right) \cap E .
$$

If either (i) $d=1$ or (ii) $d=2, g=0$ and the contact vector of $f$ with $V_{0}$ is (2), then the image of $f$ lies in $E$.

Fix a neighborhood $U$ of the zero section $E$ whose closure is disjoint from the infinity section of $\mathbb{F}_{1}$. For $V$-compatible $(J, \nu)$ and ordered sequences $s^{1}$ and $s^{2}$ with $\operatorname{deg}\left(s^{1}\right)=\operatorname{deg}\left(s^{2}\right)=2$, choose $\ell\left(s^{1}\right)$ points $\left\{p_{i}\right\}$ in $V_{1}$ and $\ell\left(s^{2}\right)$ points $\left\{q_{j}\right\}$ in $V_{2}$ and let

$$
\mathcal{M}_{0, s^{1},(2), s^{2}}^{V_{1}, V_{0}, V_{2}}(U, 2 S, J, \nu) \cap\left\{p_{i}, q_{j}\right\} \subset \mathcal{M}_{0, s^{1},(2), s^{2}}^{V_{1}, V_{0}, V_{2}}(U, 2 S, J, \nu)
$$

denote the cut-down moduli space of $V$-regular maps $f$ into $U$ with a (connected) domain of genus zero and with $n$ contact points $\left\{x_{i}\right\}$ satisfying: $f\left(x_{0}\right) \in V_{0}, f\left(x_{i}\right)=$ $p_{i}$ and $f\left(x_{\ell\left(s^{1}\right)+j}\right)=q_{j}$, where $n=\ell\left(s^{1}\right)+\ell\left(s^{2}\right)+1$. This cut-down moduli space has (formal) dimension zero. Consider a sequence of maps $\left(f_{k}, C_{k} ;\left\{x_{i}^{k}\right\}\right)$ in the cut-down moduli spaces

$$
\mathcal{M}_{0, s^{1},(2), s^{2}}^{V_{1}, V_{0}, V_{2}}\left(U, 2 S, J_{k}, \nu_{k}\right) \cap\left\{p_{i}^{k}, q_{j}^{k}\right\},
$$

where the points $\left\{p_{i}^{k}\right\} \sqcup\left\{q_{j}^{k}\right\} \subset\left(V_{1} \sqcup V_{2}\right)$ converge to points in $\left(V_{1} \sqcup V_{2}\right) \cap E$ and $V$-compatible $\left(J_{k}, \nu_{k}\right)$ converges to the complex structure of $\mathbb{F}_{1}$ as $k \rightarrow \infty$. By the Gromov Compactness Theorem, after passing to subsequences, the sequence of maps $\left(f_{k}, C_{k} ;\left\{x_{i}^{k}\right\}\right)$ then converges to a holomorphic map

$$
\left(f, C ;\left\{x_{i}\right\}\right) \in \overline{\mathcal{M}}_{0, n}(\bar{U}, 2 S) .
$$

Since $\operatorname{Im}(f) \subset \bar{U}$, every component of $C$ mapped entirely into $V$ is a ghost component. The following lemma shows that the image of $f$ lies in the zero section E. Let $s^{0}=(2)$. 
Lemma 6.4. Let $\left(f, C ;\left\{x_{i}\right\}\right)$ be as above and let $C_{i}$ denote an irreducible component $C$ that contains a marked point $x_{i_{0}}$ mapped into $V_{i}$. If $s^{i}=(2)$ for some $0 \leq i \leq 2$, then we have

(a) if $f$ is $V_{i}$-regular, then the restriction $f$ to $C_{i}$ represents the class $2 S$,

(b) if $f$ is not $V_{i}$-regular, then $C_{i}$ is a ghost component with $x_{j} \notin C_{i}$ for $j \neq i_{0}$ and $C \backslash C_{i}$ has two connected components $C_{i}^{\ell}$ such that the restriction of $f$ to $C_{i}^{\ell}$ represents the class $S$.

In particular, the image of $f$ lies in the zero section $E$ of $\mathbb{F}_{1}$.

Proof. First note that, since $\operatorname{Im}\left(f_{k}\right) \rightarrow \operatorname{Im}(f)$ and $\operatorname{Im}(f) \subset \bar{U}$, we have

(i) $\operatorname{Im}(f) \cap\left(V_{1} \sqcup V_{2}\right)=\left(V_{1} \sqcup V_{2}\right) \cap E$,

(ii) the restriction of $f$ to each component of $C$ represents the class $k S$ where $0 \leq k \leq 2$.

If a marked point $x_{i}$ is a limit point of the contact points $x_{i}^{k}$ of $f_{k}$ with $V_{i}$, i.e. $f_{k}\left(x_{i}^{k}\right) \in V_{i}$, then $x_{i}$ is a contact point of $f$ with the same contact order as $x_{i}^{k}$ unless a component containing $x_{i}$ maps into $V$. Thus, if $f$ is $V_{i}$-regular, then $f$ has a contact vector (2) with $V_{i}$ at $x_{i_{0}}$, and hence by (ii) the restriction of $f$ to $C_{i}$ represents the class $2 S$. This proves (a).

On the other hand, if $f$ is not $V_{i}$-regular, then $C_{i}$ is a ghost component mapped into $V_{i}$. Moreover, by the assumption $s^{i}=(2)$ only one marked point $x_{i_{0}}$ maps into $V_{i}$, so $x_{j} \notin C_{i}$ for $j \neq i_{0}$. Note that, since $C$ is a connected curve of (arithmetic) genus zero, each irreducible component of $C$ is smooth of genus zero. Thus Remark 1.1 implies that $C \backslash C_{i}$ has at least two connected components. Let $C_{i}^{\ell}$ be a connected component of $C \backslash C_{i}$. Then

$$
\left|C_{i}^{\ell} \cap \overline{C \backslash C_{i}^{\ell}}\right|=\left|C_{i}^{\ell} \cap C_{i}\right|=1,
$$

where the second equality follows from the fact $C$ is a connected curve of genus zero. So, if $C_{i}^{\ell}$ maps to a point, then $f\left(C_{i}^{\ell}\right) \in V_{i}$ and, by Remark 1.1. $C_{i}^{\ell}$ has at least two marked points $x_{j}$ with $f\left(x_{j}\right) \in V_{i}$. This is impossible since $f\left(x_{j}\right) \in V_{j}$ for some $V_{j}$ disjoint with $V_{i}$. Thus there are two connected components of $C \backslash C_{i}$ such that the restriction of $f$ to both components represent the class $S$. This completes the proof (b).

Now (a), (b), (i), (ii) and Lemma 6.3 imply that the image of $f$ lies in the zero section $E$ since $s^{0}=(2)$.

Remark 6.5. Let $f$ be a limit map as in Lemma 6.4 and suppose that $C_{0}$ and $C_{1}$ are irreducible components of the domain $C$ of $f$ which contain marked points $x_{0}$ and $x_{1}$ mapped into $V_{0}$ and $V_{1}$. Suppose $s^{1}=(2)$. Then, since $s^{0}=(2)$, Lemma 6.4 implies that $f$ is $V_{0}$-regular if and only if $f$ is $V_{1}$-regular. Suppose $f$ is not $\left(V_{0} \sqcup V_{1}\right)$-regular. Then, by Lemma 6.4(b), both $C_{0}$ and $C_{1}$ are ghost components with $C_{0} \cap C_{1}=\emptyset$. Since the domain $C$ is a connected curve of genus zero, the same argument for the proof of Lemma 6.4(b) shows that $C \backslash\left(C_{0} \sqcup C_{1}\right)$ has at least three connected components $C_{01}^{\ell}$ such that the restriction of $f$ to each $C_{01}^{\ell}$ represents the class $S$. This is impossible, so (i) $f$ must be $\left(V_{0} \sqcup V_{1}\right)$-regular, (ii) $C_{0}=C_{1}$, and (iii) the restriction of $f$ to the component $C_{0}=C_{1}$ represents the class $2 S$. In particular, if $s^{1}=s^{2}=(2)$, then $f^{-1}(V)$ consists of three contact points. This contradicts Remark 6.2. Therefore, if $s^{1}=s^{2}=(2)$, then for all large $k$ the cut-down moduli space (6.2) must be empty. 
For $V$-compatible $(J, \nu)$ and for points $\left\{p_{i}\right\}$ in $V_{1}$ and $\left\{q_{j}\right\}$ in $V_{2}$, denote by

$$
\mathcal{M}=\mathcal{M}_{0, s^{1},(2), s^{2}}^{V_{1}, V_{0}, V_{2}}\left(\mathbb{F}_{1}, 2 S\right) \cap\left\{p_{i}, q_{j}\right\}
$$

the cut-down moduli space of $V$-regular $(J, \nu)$-holomorphic maps into $\mathbb{F}_{1}$ with the point constraints $\left\{p_{i}, q_{j}\right\}$. Then we have a splitting of contributions to relative $\mathrm{GW}$ invariant

$$
G W_{\mathbb{F}_{1}, 2 S, 0, s_{1},(2), s_{2}}^{V_{1}, V_{0}, V_{2}}\left(C_{p t^{\ell\left(s^{1}\right)}}, C_{F}, C_{p t^{\ell\left(s^{2}\right)}}\right)=[\mathcal{M}]=[\mathcal{M}(U)]+[\mathcal{M} \backslash \mathcal{M}(U)]
$$

where $\mathcal{M}(U)$ is the cut-down moduli space (6.1) and $\mathcal{M} \backslash \mathcal{M}(U)$ consists of maps $f$ in $\mathcal{M}$ whose image does not lie in $U \subset \mathbb{F}_{1}$. Here, $[\cdot]$ denotes the zero-dimensional homology class defined by cut-down moduli space as in Remark 3.4. In general, this splitting is not well-defined, namely it depends on the choice of $(J, \nu)$ and the point constraints $\left\{p_{i}, q_{j}\right\}$. However, by the Gromov Compactness Theorem and Lemma 6.4, the splitting (6.4) is well-defined whenever (i) $(J, \nu)$ is close to the complex structure of $\mathbb{F}_{1}$ and (ii) points $\left\{p_{i}, q_{j}\right\}$ are also close to points in $\left(V_{1} \sqcup V_{2}\right) \cap E$. In such cases, the contribution

$$
[\mathcal{M}(U)]=\left[\mathcal{M}_{0, s^{1},(2), s^{2}}^{V_{1}, V_{0}, V_{2}}\left(U, 2 S ; C_{p t^{\ell\left(s^{1}\right)}}, C_{F}, C_{p t^{\ell\left(s^{2}\right)}}\right)\right]
$$

is independent of the choice of $(J, \nu)$ and $\left\{p_{i}, q_{j}\right\}$. Furthermore, this local contribution is independent of the choice of the neighborhood $U$ of the zero section $E$ in $\mathbb{F}_{1}$.

Lemma 6.6. For any neighborhood $U$ of the zero section $E$ in $\mathbb{F}_{1}$ whose closure is disjoint from the infinity section of $\mathbb{F}_{1}$, we have

$$
\begin{gathered}
\text { (a) }\left[\mathcal{M}_{0,(2),(2),(2)}^{V_{1}, V_{0}, V_{2}}\left(U, 2 S ; C_{p t}, C_{F}, C_{p t}\right)\right]=0, \\
\text { (b) }\left[\mathcal{M}_{0,(2),(2),(1,1)}^{V_{1}, V_{0}, V_{2}}\left(U, 2 S ; C_{p t}, C_{F}, C_{p t^{2}}\right)\right]=1 .
\end{gathered}
$$

Proof. (a) follows from Remark 6.5. Let $s^{1}=(2), s^{2}=(1,1)$ and $\left(f, C ;\left\{x_{i}\right\}\right)$ be a limit map as in 6.3). Again by Remark 6.5. $f$ is a $\left(V_{1} \sqcup V_{0}\right)$-regular map into $E \subset \mathbb{F}_{1}$ and the restriction of $f$ to the component $C_{0}=C_{1}$ containing $x_{0}$ and $x_{1}$ represents the class $2 S$. The stability of $f$ then implies either $C=C_{0}$ or $C=C_{0} \cup C_{2}$, where $C_{2}$ is a ghost component containing the marked points $x_{2}$ and $x_{3}$. Suppose $C=C_{0} \cup C_{2}$. In this case, $C$ has one node since the (arithmetic) genus of $C$ is zero. The restriction $f_{0}=f_{\mid C_{0}}$ has a contact order two with $V_{2}$ at the node of $C$, so $f_{0}^{-1}(V)$ consists of three contact points. This contradicts Remark 6.2. Therefore, $f$ is a holomorphic map from $C=\mathbb{P}^{1}$ into $E=\mathbb{P}^{1}$ of degree two with two ramification points $x_{0}$ and $x_{1}$ and $f\left(x_{i}\right) \in V_{2} \cap E$ for $i=2,3$. Observe that there is a unique such map $f$.

Let $J$ denote the complex structure on $\mathbb{F}_{1}$ and set

$$
\mathcal{M}^{V}=\mathcal{M}_{0,(2),(2),(1,1)}^{V_{1}, V_{0}, V_{2}}(U, 2 S, J) \text { and } h=h_{(2)} \times h_{(1,1)}: \mathcal{M}^{V} \rightarrow V_{1} \times\left(V_{2} \times V_{2}\right),
$$

where $h_{(2)}$ and $h_{(1,1)}$ are evaluation maps as in (3.2). Let $D_{f}$ be the (full) linearization of the holomorphic map equation at $f$. Since the normal bundle of $\operatorname{Im}(f)=E$ is $\mathcal{O}_{E}(1)$, we have

- $\operatorname{coker} D_{f}=H^{1}\left(f^{*} \mathcal{O}_{E}(1)\right)=H^{1}\left(\mathcal{O}_{\mathbb{P}^{1}}(2)\right)=0$, and hence

- $\mathcal{M}^{V}$ is smooth near $f$ with $T_{f} \mathcal{M}^{V}=H^{0}\left(f^{*} \mathcal{O}_{E}(1)\right)=H^{0}\left(\mathcal{O}_{\mathbb{P}^{1}}(2)\right)$,

- $d h_{f}(\xi)=\left(\xi\left(x_{1}\right), \xi\left(x_{2}\right), \xi\left(x_{3}\right)\right)$. 
In fact, regarding the neighborhood $U$ of $E$ in $\mathbb{F}_{1}$ as a disk subbundle of $\mathcal{O}_{E}(1)$, one can identify holomorphic sections $\xi$ of $f^{*} U \subset f^{*} \mathcal{O}_{E}(1)$ with $V$-regular holomorphic maps $f_{\xi}$ in $\mathcal{M}^{V}$ - in local trivialization, $f_{\xi}(x)=(f(x), \xi(x))$. By Remark 6.1 the differential $d h_{f}$ is one-to-one. Thus $d h_{f}$ is onto since $h^{0}\left(\mathcal{O}_{\mathbb{P}^{1}}(2)\right)=3$. We can now conclude that the contribution of $\left(f, \mathbb{P}^{1} ;\left\{x_{i}\right\}\right)$ to the invariant (6.5) is +1 since $f$ has no nontrivial automorphisms and $d h_{f}$ is onto and complex linear. This completes the proof of (b).

We will compute the local contribution (6.5) for the case $s^{1}=s^{2}=(1,1)$ in the next section (see (7.10) ).

\section{Spin CURVE DEgeneration AND The SUm FORMUla I}

This section proves Theorem B (a) in three steps. First we review the dualizing sheaf. The dualizing sheaf $\omega_{X}$ of a variety $X$ (if exists) is the unique invertible sheaf making Serre duality valid; when $X$ is smooth, $\omega_{X}$ is the canonical bundle $K_{X}$. For a proper holomorphic map $f: X \rightarrow B$ between two smooth varieties, the relative dualizing sheaf $\omega_{f}$ is the locally free rank one sheaf $\omega_{X} \otimes\left(f^{*} \omega_{B}\right)^{-1}$ whose restriction to each fiber $X_{b}$ is the dualizing sheaf of $X_{b}$ (cf. [T] and [HM]).

Step 1. Let $D_{1} \cup_{q} D_{2}$ be a union of two smooth curves $D_{1}$ and $D_{2}$ of genera $h_{1}$ and $h_{2}$, meeting at one point $q$. Blowing up the point $q$ yields a nodal curve $D_{0}$ with an exceptional component $E=\mathbb{P}^{1}$ that meets $\bar{D}=D_{1} \sqcup D_{2}$ at two points. A theta characteristic of the nodal curve $D_{0}$ is a line bundle $N_{0}$ together with a homomorphism $\phi: N_{0}^{2} \rightarrow \omega_{D_{0}}$ satisfying:

- $N_{0}$ restricts to $\mathcal{O}(1)$ on the exceptional component $E$,

- $\phi$ vanishes identically on $E$ and restricts to an isomorphism $\left.N_{0}^{2}\right|_{\bar{D}} \simeq \omega_{\bar{D}}$,

where $\omega_{D_{0}}$ and $\omega_{\bar{D}}$ are the dualizing sheaves of $D_{0}$ and $\bar{D}$ respectively. Since $\omega_{\bar{D}}$ is the canonical bundle of $\bar{D}$, the line bundle $N_{0}$ restricts to theta characteristic $N_{1}$ on $D_{1}$ and restricts to theta characteristic $N_{2}$ on $D_{2}$. The triple $\left(D_{0}, N_{0}, \phi\right)$ is a spin curve of genus $h=h_{1}+h_{2}$ with parity $p \equiv p_{1}+p_{2}(\bmod 2)$, where $p_{i}$ is a parity of the spin curve $\left(D_{i}, N_{i}\right)$. It then follows from a universal deformation of the spin curve $\left(D_{0}, N_{0}, \phi\right)$ (cf. p. $570[\mathrm{C}$ ) that there are

- a family of curves $\rho: \mathcal{D} \rightarrow \Delta$, where $\Delta$ is a unit disk in $\mathbb{C}$, the fiber $D_{\lambda}$ over $\lambda \neq 0$ is a smooth curve of genus $h$ and the central fiber is the nodal curve $D_{0}$,

- a line bundle $\pi: \mathcal{N} \rightarrow \mathcal{D}$ together with a homomorphism $\Phi: \mathcal{N}^{2} \rightarrow \omega_{\rho}$ such that each $\left(\mathcal{D}_{\mid \rho^{-1}(\lambda)}, \mathcal{N}_{\mid \rho^{-1}(\lambda)}, \Phi_{\mid \rho^{-1}(\lambda)}\right)$ is a spin curve of genus $h$ with parity $p$,

where $\omega_{\rho}$ is the relative dualizing sheaf of $\rho$ (for more details see $[\mathbf{C}$ ).

Let $\mathcal{N}_{D_{\lambda}}(\lambda \neq 0)$ be the total space of $\mathcal{N}_{\mid D_{\lambda}}$ and $\mathcal{N}_{\bar{D}}$ be the total space of $\mathcal{N}_{\mid \bar{D}}$. Since both total spaces $\mathcal{N}_{D_{\lambda}}$ and $\mathcal{N}_{\bar{D}}$ are smooth, there are short exact sequences

$0 \rightarrow \pi^{*} \mathcal{N}_{\mid D_{\lambda}} \rightarrow T \mathcal{N}_{D_{\lambda}} \rightarrow \pi^{*} T D_{\lambda} \rightarrow 0 \quad$ and $\quad 0 \rightarrow \pi^{*} \mathcal{N}_{\mid \bar{D}} \rightarrow T \mathcal{N}_{\bar{D}} \rightarrow \pi^{*} T \bar{D} \rightarrow 0$.

It then follows from these exact sequences that

$$
K_{\mathcal{N}_{D_{\lambda}}}=\pi^{*} \mathcal{N}_{\mid D_{\lambda}}^{*} \otimes \pi^{*} K_{D_{\lambda}} \quad \text { and } \quad K_{\mathcal{N}_{\bar{D}}}=\pi^{*} \mathcal{N}_{\mid \bar{D}}^{*} \otimes \pi^{*} K_{\bar{D}}
$$


On the other hand, the homomorphism $\Phi: \mathcal{N}^{2} \rightarrow \omega_{\rho}$ induces a homomorphism

$$
\Phi^{\prime}: \pi^{*} \mathcal{N}=\pi^{*}\left(\mathcal{N}^{*} \otimes \mathcal{N}^{2}\right) \longrightarrow \pi^{*}\left(\mathcal{N}^{*} \otimes \omega_{\rho}\right) .
$$

Let $\sigma$ be the tautological section of $\pi^{*} \mathcal{N}$. Since the relative dualizing sheaf $\omega_{\rho}$ restricts to dualizing sheaf $\omega_{D_{\lambda}}$ on $D_{\lambda}$, by (7.1) the composition $\Phi^{\prime} \circ \sigma$ is a section of $\pi^{*}\left(\mathcal{N}^{*} \otimes \omega_{\rho}\right)$ satisfying:

- $\Phi^{\prime} \circ \sigma$ restricts to a holomorphic 2 -form $\alpha_{\lambda}$ on $\mathcal{N}_{D_{\lambda}}(\lambda \neq 0)$ whose zero set is $D_{\lambda}$,

- $\Phi^{\prime} \circ \sigma$ restricts to a holomorphic 2-form $\alpha_{0}$ on $\mathcal{N}_{\bar{D}}$ whose zero set is $\bar{D}$.

Let $\mathcal{N}_{E}$ denote the total space of $\mathcal{N}_{\mid E}$. For sufficiently small $\epsilon>0$, choose a bump function $\beta$ that is 1 on the complement of the $2 \epsilon$-neighborhood of $\mathcal{N}_{E}$ in $\mathcal{N}_{\mathcal{D}}$ and vanishes on the $\epsilon$-neighborhood $\mathcal{U}_{\epsilon}$ of $\mathcal{N}_{E}$. For each point $x \in \mathcal{N}_{\mathcal{D}} \backslash \mathcal{U}_{\epsilon}$ we set $\beta \alpha_{\lambda}\left(v_{x}, \cdot\right)=0$ for normal vectors $v_{x}$ to submanifold $\mathcal{N}_{D_{\lambda}}$ (or $\mathcal{N}_{\bar{D}}$ for $\lambda=0$ ) at $x$ and define $\alpha=\beta \alpha_{\lambda}$ at $x$. Extension $\alpha$ by zero then gives a 2 -form on $\mathcal{N}_{\mathcal{D}}$ that restricts to $\beta \alpha_{\lambda}$ on $\mathcal{N}_{D_{\lambda}}(\lambda \neq 0)$ and to $\beta \alpha_{0}$ on $\mathcal{N}_{\bar{D}}$.

Step 2. Consider the projectivization $\mathbb{P}\left(\mathcal{N} \oplus \mathcal{O}_{\mathcal{D}}\right)$ over $\mathcal{D}$ that gives a degeneration

$$
\lambda: Z=\mathbb{P}\left(\mathcal{N} \oplus \mathcal{O}_{\mathcal{D}}\right) \rightarrow \mathcal{D} \rightarrow \Delta
$$

whose fiber $Z_{\lambda}(\lambda \neq 0)$ is a ruled surface over $D_{\lambda}$ isomorphic to $\mathbb{P}_{h}=\mathbb{P}\left(N \oplus \mathcal{O}_{D}\right)$, where $(D, N)$ is a (smooth) spin curve of genus $h=h_{1}+h_{2}$ with parity $p \equiv p_{1}+p_{2}$ $(\bmod 2)$ and whose central fiber $Z_{0}$ is the singular (ruled) surface

$$
\mathbb{P}_{h_{1}} \cup_{V_{1}} \mathbb{F}_{1} \cup_{V_{2}} \mathbb{P}_{h_{2}} \rightarrow D_{0},
$$

where $V_{1}$ and $V_{2}$ are fibers over the nodes of $D_{0}$. Note that the general fiber $Z_{\lambda}$ $(\lambda \neq 0)$ is the symplectic fiber sum

$$
\mathbb{P}_{h}=\mathbb{P}_{h_{1}} \# V_{1} \# \mathbb{F}_{1} \# V_{V_{2}} \mathbb{P}_{h_{2}} .
$$

Let $U$ be an (open) neighborhood of the zero section of $Z=\mathbb{P}\left(\mathcal{N} \oplus \mathcal{O}_{\mathcal{D}}\right)$ and fix an isomorphism $\Psi$ from $U$ to some neighborhood of $\mathcal{D} \subset \mathcal{N}_{\mathcal{D}}$ taking the zero section of $Z$ to $\mathcal{D}$. Choose a point $q_{0}$ in the exceptional component $E \subset D_{0}$ that is not a nodal point and let $B \subset \mathcal{D}$ be a normal disk to $E \subset \mathcal{D}$ at $q_{0}$, namely the intersection $B \cap D_{\lambda}$ is one point for all small $|\lambda|$. Let $\tilde{V}_{\lambda}$ be the fiber of $Z_{\lambda} \rightarrow D_{\lambda}$ over the intersection point $B \cap D_{\lambda}$ and set

$$
V=V_{1} \sqcup V_{0} \sqcup V_{2} \text {, }
$$

where $V_{0}=\tilde{V}_{0}$. Denote by $\mathcal{J}(Z)$ the space of all $(J, \nu)$ on $Z$ satisfying: (i) each $Z_{\lambda}$ is $J$-invariant and (ii) the restriction of $(J, \nu)$ to $Z_{0}$ and $Z_{\lambda}(\lambda \neq 0)$ are $V$ compatible and $\tilde{V}_{\lambda}$-compatible respectively. We will use the same notation $(J, \nu)$ for its restriction to each $Z_{\lambda}$. Denote by the same $S$ the section classes of $\mathbb{P}_{h_{1}}, \mathbb{F}_{1}$, $\mathbb{P}_{h_{2}}$ and $Z_{\lambda}$ represented by the zero sections. Let $D_{i}(i=1,2)$ and $D_{\lambda}$ denote the zero sections of $\mathbb{P}_{h_{i}}$ and $Z_{\lambda}$ respectively. For each small $|\lambda|$ we set

$$
U_{\lambda}=U \cap Z_{\lambda} .
$$

By using the 2-form $\alpha$ on $\mathcal{N}_{D}$ together with the isomorphism $\Psi$, we obtain:

Lemma 7.1. There is an almost complex structure $J_{V}$ on $Z$ satisfying:

(a) $\left(J_{V}, 0\right) \in \mathcal{J}(Z)$ and $J_{V}$ restricts to the complex structure of $\mathbb{F}_{1}$,

(b) $\overline{\mathcal{M}}_{\chi, n}^{*}\left(U_{\lambda}, d S, J_{V}\right)=\overline{\mathcal{M}}_{\chi, n}^{*}\left(D_{\lambda}, d\right) \quad$ and $\quad \overline{\mathcal{M}}_{\chi, n}^{*}\left(\bar{U}_{0} \cap \mathbb{P}_{h_{i}}, d S, J_{V}\right)=$ $\overline{\mathcal{M}}_{\chi, n}^{*}\left(D_{i}, d\right)$, 
(c) for generic $(J, \nu) \in \mathcal{J}(Z)$ sufficiently close to $\left(J_{V}, 0\right)$ and for small $|\lambda|>0$, $\left[\mathcal{M}_{\chi,(2)}^{\tilde{V}_{\lambda}, *}\left(U_{\lambda}, 2 S\right)\right]=G T_{(2)}^{l o c, h, p} \quad$ and $\quad\left[\mathcal{M}_{\chi_{i}, s_{i}}^{V_{i}, *}\left(\mathbb{P}_{h_{i}} \cap U_{0}, 2 S\right)\right]=G T_{s_{i}}^{l o c, h_{i}, p_{i}}$, where $\chi=2-4 h, \chi_{i}=2 \ell\left(s_{i}\right)-4 h_{i}$ for $i=1,2$.

Proof. By the isomorphism $\Psi$ as above, one can regard $\alpha_{\lambda}(\lambda \neq 0)$ and $\alpha_{0}$ as holomorphic 2-forms on $U_{\lambda}$ and on $U_{0} \cap\left(\mathbb{P}_{h_{1}} \sqcup \mathbb{P}_{h_{2}}\right)$ whose zero sets are $D_{\lambda}$ and $D_{1} \sqcup D_{2}$, respectively. Similarly, one can also regard $\beta$ as a bump function on $U$ and $\alpha$ as a 2 -form on $U$ satisfying (i) $\alpha$ vanishes on some neighborhoods of $\mathbb{F}_{1}$, $V$ and $\tilde{V}_{\lambda}$ for small $|\lambda|$, and (ii) the restriction of $\alpha$ to $U_{\lambda}$ and $U_{0} \cap\left(\mathbb{P}_{h_{1}} \sqcup \mathbb{P}_{h_{2}}\right)$ are respectively $\beta \alpha_{\lambda}$ and $\beta \alpha_{0}$. Now, let $J_{V}$ be the almost complex structure on $Z$ induced by $\alpha$ and the formula (0.1). Then, (a) follows from (i), (b) follows from (ii) and Remark 2.2 and (c) follow from definition and compactness by (b).

Remark 7.2. Let $f$ be a map in $\mathcal{M}_{\chi,(2)}^{\tilde{V}_{\lambda}}\left(U_{\lambda}, 2 S\right)$ with $f^{-1}\left(\tilde{V}_{\lambda}\right)=\{x\}$, where the Euler characteristic $\chi=2-4 h$. If there is a connected component of the domain of $f$ that does not contain the contact point $x$, then the restriction of $f$ to that component represents the trivial homology class. The stability of $f$ thus shows $C$ is connected since there are no marked points except the contact point $x$. Consequently, for $\lambda \neq 0$ we have

$$
\mathcal{M}_{\chi,(2)}^{\tilde{V}_{\lambda}, *}\left(U_{\lambda}, 2 S\right)=\mathcal{M}_{\chi,(2)}^{\tilde{V}_{\lambda}}\left(U_{\lambda}, 2 S\right)=\mathcal{M}_{g,(2)}^{\tilde{V}_{\lambda}}\left(U_{\lambda}, 2 S\right)
$$

where the genus $g=2 h$. Similar arguments also show that

$$
\mathcal{M}_{\chi_{0}, s_{1},(2), s_{2}}^{V_{1}, V_{0}, V_{2}, *}\left(\mathbb{F}_{1}, 2 S\right)=\mathcal{M}_{g_{0}, s_{1},(2), s_{2}}^{V_{1}, V_{0}, V_{2}}\left(\mathbb{F}_{1}, 2 S\right)
$$

where the Euler characteristic $\chi_{0}=2$ and the genus $g_{0}=0$.

Step 3. Choose an (open) neighborhood $W$ of the zero section of $Z=\mathbb{P}\left(\mathcal{N} \oplus \mathcal{O}_{\mathcal{D}}\right)$ with $\bar{W} \subset U$, and for each small $|\lambda|$ set

$$
W_{\lambda}=W \cap Z_{\lambda} .
$$

The following is the key fact to the proof of Theorem B (a).

Lemma 7.3. For $(J, \nu) \in \mathcal{J}(Z)$ sufficiently close to $\left(J_{V}, 0\right)$ and for small $|\lambda|>0$, we have

$$
\mathcal{M}_{\chi,(2)}^{\tilde{V}_{\lambda}}\left(U_{\lambda}, 2 S\right) \backslash \mathcal{M}_{\chi,(2)}^{\tilde{V}_{\lambda}}\left(W_{\lambda}, 2 S\right)=\emptyset
$$

where the Euler characteristic $\chi=2-4 h$.

Proof. Suppose not. Then, there exists a sequence of $\left(J_{k}, \nu_{k}\right)$-holomorphic maps $f_{k}$ into $U_{\lambda_{k}}$ with $\operatorname{Im}\left(f_{k}\right) \cap\left(U_{\lambda_{k}} \backslash W_{\lambda_{k}}\right) \neq \emptyset$, where $\lambda_{k} \rightarrow 0$ and $\left(J_{k}, \nu_{k}\right)$ converges to $\left(J_{V}, 0\right)$ as $k \rightarrow \infty$. After passing to subsequences, by the Gromov Compactness Theorem, $f_{k}$ converges to a $J_{V}$-holomorphic map $f$ into $Z_{0}$ such that (i) $\operatorname{Im}(f) \subset$ $\bar{U}_{0}$ and (ii) $\operatorname{Im}(f) \cap\left(\bar{U}_{0} \backslash W_{0}\right) \neq \emptyset$. By (i) and Lemma 7.1, $f$ can be split as $f=\left(f_{1}, f_{0}, f_{2}\right)$, where $f_{1}$ and $f_{2}$ are respectively holomorphic maps into $D_{1}$ and $D_{2}$, and $f_{0}$ is a holomorphic map into $\mathbb{F}_{1}$ such that

$$
\operatorname{Im}\left(f_{0}\right) \cap\left(V_{1} \sqcup V_{2}\right)=\left(\operatorname{Im}\left(f_{1}\right) \sqcup \operatorname{Im}\left(f_{2}\right)\right) \cap\left(V_{1} \sqcup V_{2}\right)=E \cap\left(V_{1} \sqcup V_{2}\right) .
$$

Note that the domain $C$ of $f$ is a connected curve of genus $2 h$ since by (7.2) $f$ is a limit of maps with connected domains of genus $2 h$. Also note that if $f$ is not $\left(V_{1} \sqcup V_{2}\right)$-regular, there is a ghost component mapped into $V_{1} \sqcup V_{2}$. 
Let $f_{12}=\left(f_{1}, f_{2}\right), C_{12}$ be the domain of $f_{12}$ and $C_{0}$ be the domain of $f_{0}$. We can assume that $C_{0}$ contains all ghost components mapped into $V_{1} \sqcup V_{2}$. Then $f_{12}$ is $\left(V_{1} \sqcup V_{2}\right)$-regular, so $f_{12}^{-1}\left(V_{1} \sqcup V_{2}\right)=\left(C_{12} \cap C_{0}\right)$. Let $\ell=\left|f_{12}^{-1}\left(V_{1} \sqcup V_{2}\right)\right|$. Since $g-1=-\frac{1}{2} \chi$, we have

$$
2 h=g(C)=-\frac{1}{2} \chi\left(C_{0}\right)-\frac{1}{2} \chi\left(C_{12}\right)+\ell+1 .
$$

Consider $f_{12}$ as a holomorphic map into $D_{1} \sqcup D_{2}$ and apply the Riemann-Hurwitz formula to each irreducible component of $C_{12}$. This gives

$$
-\frac{1}{2} \chi\left(C_{12}\right)+\ell \geq 2 h,
$$

since the geometric genus of each irreducible component is less than or equal to its arithmetic genus and $f_{12}$ has at least $2(4-\ell)$ ramification points. Consequently, by (7.5) and (7.6) we have

$$
\chi\left(C_{0}\right) \geq 2 .
$$

Note that the image of $f_{0}$ does not lie in the zero section $E$ of $\mathbb{F}_{1}$ by (ii) since $f_{12}$ maps into $D_{1} \cup D_{2} \subset W_{0}$. Remark 6.1 and (7.4) imply that there is exactly one irreducible component $C_{0}^{\prime}$ of $C_{0}$ such that the restriction $f_{0}^{\prime}=f_{\mid C_{0}^{\prime}}$ has contact vectors (2) with $V_{1}$ and $V_{2}$ and all other irreducible components of $C_{0}$ are ghost components. Let $C_{0}^{1}$ be the connected component of $C_{0}$ that contains $C_{0}^{\prime}$. Since $f$ has no degree zero components, we have

- all ghost components mapped into $V_{0}$ are contained in $C_{0}^{1}$,

- if there exists a connected component $C_{0}^{2} \neq C_{0}^{1}$ of $C_{0}$, then $C_{0}^{2}$ is a union of ghost components such that $C_{0}^{2}$ has no marked points and maps into either $V_{1}$ or $V_{2}$. Since $\left|C_{0} \cap C_{12}\right|=\ell \leq 4$ and $C_{0}^{1} \cap C_{12}$ contains at least one point mapped into $V_{1}$ and at least one point mapped into $V_{2}$, we have $\left|C_{0}^{2} \cap \overline{C \backslash C_{0}^{2}}\right|=\left|C_{0}^{2} \cap C_{12}\right|=1$, and hence $g\left(C_{0}^{2}\right)>0$ by Remark 1.1]

Now from (7.7) we have $g\left(C_{0}^{1}\right)=0$, so each irreducible component of $C_{0}^{1}$ has genus zero and no two irreducible components meet at more than one point. In particular, since $C_{0}^{\prime}$ has genus zero, Lemma 6.3 implies that $f_{0}^{\prime}$ has a contact vector $(1,1)$ with $V_{0}$. In this case, since $f$ is a limit map of a sequence of maps with contact order (2) with $V_{0}$, there is a ghost component mapped into $V_{0}$. Let $C_{0}^{\prime \prime}$ be a connected component of the union of all ghost components mapped into $V_{0}$. Then, $C_{0}^{\prime \prime} \subset C_{0}^{1}$, so $g\left(C_{0}^{\prime \prime}\right)=0$ and

$$
\left|C_{0}^{\prime \prime} \cap \overline{C \backslash C_{0}^{\prime \prime}}\right|=\left|C_{0}^{\prime \prime} \cap C_{0}^{\prime}\right|<2 .
$$

Since $C_{0}^{\prime \prime}$ has at most one marked point, we have a contradiction by Remark 1.1 .

Proof of Theorem B (a). The proof is identical to the proof of Theorem A. We only outline the proof. For (ordered) sequences $s^{i}$ with $\operatorname{deg}\left(s_{i}\right)=2$ where $i=1,2$, consider the evaluation map that records the intersection points with $V_{1}$ and $V_{2}$ :

$$
\begin{aligned}
e v_{s^{1}, s^{2}, U_{0}}^{*}: & \cup \mathcal{M}_{\chi_{1}, s^{1}}^{V_{1},}\left(\mathbb{P}_{h_{1}} \cap U_{0}, 2 S\right) \times \mathcal{M}_{\chi_{0}, s^{1},(2), s^{2}}^{V_{1}, V_{0}, V_{2}, *}\left(\mathbb{F}_{1} \cap U_{0}, 2 S\right) \\
& \times \mathcal{M}_{\chi_{2}, s^{2}}^{V_{2}, *}\left(\mathbb{P}_{h_{2}} \cap U_{0}, 2 S\right) \\
& \longrightarrow\left(V_{1}^{\ell\left(s^{1}\right)} \times V_{1}^{\ell\left(s^{1}\right)}\right) \times\left(V_{2}^{\ell\left(s^{2}\right)} \times V_{2}^{\ell\left(s^{2}\right)}\right),
\end{aligned}
$$

where the union is over all $\chi_{1}+\chi_{0}+\chi_{2}-2 \ell\left(s^{1}\right)-2 \ell\left(s^{2}\right)=2-4 h$. Let $\triangle_{s^{i}}$ be the diagonal of $V^{\ell\left(s^{i}\right)} \times V^{\ell\left(s^{i}\right)}$ for $i=1,2$. Lemma 7.3 and Theorem 10.1 of [P2 then 
give

$$
\left[\mathcal{M}_{\chi,(2)}^{\tilde{V}_{\lambda}, *}\left(U_{\lambda}, 2 S\right)\right]=\sum_{s^{1}, s^{2}} \frac{\left|s^{1}\right|\left|s^{2}\right|}{\ell\left(s^{1}\right) ! \ell\left(s^{2}\right) !}\left[\left(e v_{s^{1}, s^{2}, U_{0}}^{*}\right)^{-1}\left(\triangle_{s^{1}} \times \triangle_{s^{2}}\right)\right],
$$

where $\chi=2-4 h$. On the other hand, the splitting of the diagonal $\triangle_{s^{1}} \times \triangle_{s^{2}}$ yields

$$
\begin{aligned}
& \sum_{s^{1}, s^{2}} \frac{\left|s^{1}\right|\left|s^{2}\right|}{\ell\left(s^{1}\right) ! \ell\left(s^{2}\right) !}\left[\left(e v_{s^{1}, s^{2}, U_{0}}^{*}\right)^{-1}\left(\triangle_{s^{1}} \times \triangle_{s^{2}}\right)\right] \\
= & \sum_{m^{1}, m^{2}} \frac{\left|m^{1}\right|\left|m^{2}\right|}{m^{1} ! m^{2} !} G T_{m^{1}}^{l o c, h_{1}, p_{1}} \cdot\left[\mathcal{M}_{0, m^{1},(2), m^{2}}^{V_{1}, V_{0}, V_{2}}\left(\mathbb{F}_{1} \cap U_{0}, 2 S ; C_{p t t^{\ell\left(m^{1}\right)}}, C_{F}, C_{p t^{\ell\left(m^{2}\right)}}\right)\right] \\
& \quad G T_{m^{2}}^{l o c, h_{2}, p_{2}} \\
= & G T_{\left(1^{2}\right)}^{l o c, h_{1}, p_{1}} \cdot G T_{(2)}^{l o c, h_{2}, p_{2}}+G T_{(2)}^{l o c, h_{1}, p_{1}} \cdot G T_{\left(1^{2}\right)}^{l o c, h_{2}, p_{2}} \\
+ & \frac{1}{4} G T_{\left(1^{2}\right)}^{l o c, h_{1}, p_{1}} \cdot\left[\mathcal{M}_{0,\left(1^{2}\right),(2),\left(1^{2}\right)}^{V_{1}, V_{0}, V_{2}}\left(\mathbb{F}_{1} \cap U_{0}, 2 S ; C_{p t^{2}}, C_{F}, C_{p t^{2}}\right)\right] \cdot G T_{\left(1^{2}\right)}^{l o c, h_{2}, p_{2}},
\end{aligned}
$$

where the first equality follows from (3.4), Lemma 7.1)(c), Remark 3.1] and (7.3), and the second equality follows from Lemma 6.6. Thus, by Lemma 7.1.(c), (7.8), (7.9), Remark 5.3 and Lemma 2.6, we have

$$
\begin{aligned}
G T_{(2)}^{l o c, h, p}= & (-1)^{p_{1}} 2^{h_{1}} G T_{(2)}^{l o c, h_{2}, p_{2}}+(-1)^{p_{2}} 2^{h_{2}} G T_{(2)}^{l o c, h_{1}, p_{1}} \\
& +(-1)^{p} 2^{h-2}\left[\mathcal{M}_{0,\left(1^{2}\right),(2),\left(1^{2}\right)}^{V_{1}, V_{0}, V_{2}}\left(\mathbb{F}_{1} \cap U_{0}, 2 S ; C_{p t^{2}}, C_{F}, C_{p t^{2}}\right)\right] .
\end{aligned}
$$

When $\left(h_{2}, p_{2}\right)=(0,+)$, this equation shows

$$
\left[\mathcal{M}_{0,\left(1^{2}\right),(2),\left(1^{2}\right)}^{V_{1}, V_{0}, V_{2}}\left(\mathbb{F}_{1} \cap U_{0}, 2 S ; C_{p t^{2}}, C_{F}, C_{p t^{2}}\right)\right]=-4 G T_{(2)}^{l o c, 0,+} .
$$

This completes the proof.

\section{Spin CURVE DEgEneration AND the SUM Formula II}

This section proves Theorem B (b). Let $h \geq 2$ or $(h, p)=(1,+)$, and let $\mathcal{D} \rightarrow \Delta$ denote a family of curves over the unit disk $\Delta$ in $\mathbb{C}$ whose fiber over $\lambda \neq 0$ is a smooth curve $D_{\lambda}$ of genus $h$ and whose central fiber $D_{0}$ is a union of two smooth components $\bar{D}$ and $E$ of genera $h-1$ and 0 , meeting at two points. Fix a theta characteristic $\bar{N}$ on $\bar{D}$ with parity $p$. One can then find a line bundle $\mathcal{N} \rightarrow \mathcal{D}$ that restricts to a theta characteristic on $D_{\lambda}$ with parity $p$, to the theta characteristic $\bar{N}$ on $\bar{D}$ and to $\mathcal{O}(1)$ on $E$ (cf. p. $570\left[\mathrm{C}\right.$ ). The projectivization $\mathbb{P}\left(\mathcal{N} \oplus \mathcal{O}_{\mathcal{D}}\right)$ gives a degeneration

$$
\mathbb{P}\left(\mathcal{N} \oplus \mathcal{O}_{\mathcal{D}}\right) \rightarrow \mathcal{D} \rightarrow \Delta
$$

such that (i) the general fiber $Z_{\lambda}(\lambda \neq 0)$ is a ruled surface isomorphic to $\mathbb{P}_{h}=$ $\mathbb{P}\left(N \oplus \mathcal{O}_{D}\right)$, where $(D, N)$ is a smooth spin curve of genus $h$ with parity $p$, and (ii) the central fiber is the singular (ruled) surface

$$
\mathbb{P}_{h-1} \bigcup_{V_{1} \sqcup V_{2}} \mathbb{F}_{1} \rightarrow D_{0}
$$


where $\mathbb{P}_{h-1}=\mathbb{P}\left(\bar{N} \oplus \mathcal{O}_{\bar{D}}\right)$ and $V_{1}$ and $V_{2}$ are fibers over the nodes of $D_{0}$. Note that the general fiber $Z_{\lambda}(\lambda \neq 0)$ is the symplectic fiber sum

$$
\mathbb{P}_{h}=\mathbb{P}_{h-1} \underset{V_{1} \sqcup V_{2}}{\#} \mathbb{F}_{1} .
$$

Proof of Theorem B (b). The proof is also identical to those of Theorem A and Theorem B (a). We only sketch the proof. Fix a normal disk $B \subset \mathcal{D}$ to $E$ at some point that is not a nodal point of $D_{0}$, and for each small $|\lambda|$, let $\tilde{V}_{\lambda}$ be the fiber of $Z_{\lambda} \rightarrow D_{\lambda}$ over the intersection point of $B$ and $D_{\lambda}$. Choose small neighborhoods $U$ and $W$ of the zero section of $Z=\mathbb{P}\left(\mathcal{N} \oplus \mathcal{O}_{\mathcal{D}}\right)$ satisfying $\bar{W} \subset U$, and set

$$
W_{\lambda}=W \cap Z_{\lambda} \quad \text { and } \quad U_{\lambda}=U \cap Z_{\lambda} .
$$

The same arguments as in Lemma 7.1 and Lemma 7.3 then show that the tautological section of $\pi^{*} \mathcal{N}$ over the total space of $\pi: \mathcal{N} \rightarrow \mathcal{D}$ induces an almost complex structure $J_{V}$ on $Z$ satisfying: for $(J, \nu)$ sufficiently close to $\left(J_{V}, 0\right)$ and for small $|\lambda|>0$

$$
\begin{gathered}
G T_{(2)}^{l o c, h, p}=\left[\mathcal{M}_{\chi,(2)}^{\tilde{V}_{\lambda, *}}\left(U_{\lambda}, 2 S\right)\right] \quad \text { and } \quad G T_{s^{1}, s^{2}}^{l o c, h-1, p}=\left[\mathcal{M}_{\chi_{0}, s^{1}, s^{2}}^{V_{1}, V_{2}, *}\left(\mathbb{P}_{h-1} \cap U_{0}\right)\right], \\
(8.2) \quad \mathcal{M}_{\chi,(2)}^{\tilde{V}_{\lambda, *}}\left(U_{\lambda}, 2 S\right) \backslash \mathcal{M}_{\chi,(2)}^{\tilde{V}_{\lambda, *}}\left(W_{\lambda}, 2 S\right)=\emptyset
\end{gathered}
$$

where $\chi=2-4 h$ and $\chi_{0}=-4 h+2 \sum \ell\left(s^{i}\right)$. For ordered sequences $s^{i}$ with $\operatorname{deg}\left(s^{i}\right)=$ 2 , where $i=1,2$, consider the evaluation map that records the intersection points with $V_{1}$ and $V_{2}$ :

$$
\begin{gathered}
e v_{s^{1}, s^{2}, U_{0}}^{*}: \bigcup \mathcal{M}_{\chi_{0}, s^{1}, s^{2}}^{V_{1}, V_{2}, *}\left(\mathbb{P}_{h-1} \cap U_{0}, 2 S\right) \times \mathcal{M}_{\chi_{1}, s^{1},(2), s^{2}}^{V_{1}, V_{0}, V_{2}, *}\left(\mathbb{F}_{1} \cap U_{0}, 2 S\right) \\
\longrightarrow\left(V_{1}^{\ell\left(s^{1}\right)} \times V_{1}^{\ell\left(s^{1}\right)}\right) \times\left(V_{2}^{\ell\left(s^{2}\right)} \times V_{2}^{\ell\left(s^{2}\right)}\right)
\end{gathered}
$$

where $V_{0}=\tilde{V}_{0}$ and the union is over all $\chi_{1}+\chi_{0}-2 \ell\left(s^{1}\right)-2 \ell\left(s^{2}\right)=2-4 h$. Let $\triangle_{s^{i}}$ be the diagonal of $V^{\ell\left(s^{i}\right)} \times V^{\ell\left(s^{i}\right)}$, where $i=1,2$. Then we have

$$
\begin{aligned}
& G T_{(2)}^{l o c, h, p}=\left[\mathcal{M}_{\chi,(2)}^{\tilde{V}_{\lambda, *}}\left(U_{\lambda}, 2 S\right)\right]=\sum_{s^{1}, s^{2}} \frac{\left|s^{1}\right|\left|s^{2}\right|}{\ell\left(s^{1}\right) ! \ell\left(s^{2}\right) !}\left[\left(e v_{s^{1}, s^{2}, U_{0}}^{*}\right)^{-1}\left(\triangle_{s^{1}} \times \triangle_{s^{2}}\right)\right] \\
= & \sum_{m^{1}, m^{2}} \frac{\left|m^{1}\right|\left|m^{2}\right|}{m^{1} ! m^{2} !} G T_{m^{1}, m^{2}}^{l o c, h-1, p} \cdot\left[\mathcal{M}_{0, m^{1},(2), m^{2}}^{V_{1}, V_{0}, V_{2}}\left(\mathbb{F}_{1} \cap U_{0}, 2 S ; C_{p t^{\ell\left(m^{1}\right)}}, C_{F}, C_{p t^{\ell\left(m^{2}\right)}}\right)\right],
\end{aligned}
$$

where the first sum is over all ordered sequences $s^{1}$ and $s^{2}$ with $\operatorname{deg}\left(s^{1}\right)=\operatorname{deg}\left(s^{2}\right)=$ 2 and the second sum is over all partitions $m^{1}$ and $m^{2}$ of 2 . The first equality follows from (8.1), the second equality from (8.2) and Theorem 10.1 of [P2 and the third equality from (3.4), (8.1), Remark 3.1 and (7.3). On the other hand, by Remark 5.3 we have

$$
G T_{\left(1^{2}\right),\left(1^{2}\right)}^{l o c, h-1, p}=2^{2} G T_{2}^{l o c, h-1, p} \quad \text { and } \quad G T_{\left(1^{2}\right),(2)}^{l o c, h-1, p}=2 G T_{(2)}^{l o c, h-1, p} .
$$

This together with (8.3), Lemma 2.6. Lemma 6.6 and (7.10) completes the proof. 


\section{REDUCTION TO GENUS ZERO SPIN CURVE INVARIANTS}

As described in the Introduction, Kiem and Li proved the Maulik-Pandharipande formulas (0.2) by reducing higher genus spin curve invariants to genus zero spin curve invariants. The aim of this section is to show how their reduction follows from Theorem A and Theorem B.

Proposition 9.1 ([KL1]).

(a) $G T_{1}^{l o c, h, p}\left(\prod_{i=1}^{n} \tau_{k_{i}}\left(F^{*}\right)\right)=(-1)^{p} G T_{1}^{l o c, 0,+}\left(\prod_{i=1}^{n} \tau_{k_{i}}\left(F^{*}\right)\right)$,

(b) $G T_{2}^{l o c, h, p}\left(\prod_{i=1}^{n} \tau_{k_{i}}\left(F^{*}\right)\right)=(-1)^{p} 2^{h} G T_{2}^{l o c, 0,+}\left(\prod_{i=1}^{n} \tau_{k_{i}}\left(F^{*}\right)\right)$.

Proof. The sum formula (0.3) for $d=1,2$ and Remark 5.3 show that

$$
\begin{aligned}
G T_{1}^{l o c, h, p}\left(\prod_{i=1}^{n} \tau_{k_{i}}\left(F^{*}\right)\right) & =G T_{1}^{l o c, h, p} \cdot G T_{(1),(1)}^{\mathbb{F}_{0}}\left(\prod_{i=1}^{n} \phi_{i}^{k_{i}}\left(F^{*}\right)\right), \\
G T_{2}^{l o c, h, p}\left(\prod_{i=1}^{n} \tau_{k_{i}}\left(F^{*}\right)\right) & =\frac{1}{2} G T_{2}^{l o c, h, p} \cdot G T_{(1,1),(1,1)}^{\mathbb{F}_{0}}\left(\prod_{i=1}^{n} \phi_{i}^{k_{i}}\left(F^{*}\right)\right) \\
& +G T_{(2)}^{l o c, h, p} \cdot G T_{(2),(1,1)}^{\mathbb{F}_{0}}\left(\prod_{i=1}^{n} \phi_{i}^{k_{i}}\left(F^{*}\right)\right) .
\end{aligned}
$$

Thus, Proposition 9.1(a) follows from Lemma 2.6 and 9.1). Similarly, by Lemma 2.6 and (9.2), in order to prove Proposition 9.1(b) we need to show

$$
G T_{(2)}^{l o c, h, p}=(-1)^{p} 2^{h} G T_{(2)}^{l o c, 0,+} .
$$

The sum formula (0.4) for the case $\left(h_{2}, p_{2}\right)=(1,+)$ gives

$$
G T_{(2)}^{l o c, h, p}=(-1)^{p} 2^{h-1} G T_{(2)}^{l o c, 1,+}+2 G T_{(2)}^{l o c, h-1, p}-(-1)^{p} 2^{h} G T_{(2)}^{l o c, 0,+},
$$

where $h \geq 2$. Applying the sum formula (0.4) twice with $p_{1}=p_{2}= \pm 1$ and $h_{1}=h_{2}=1$ gives

$$
G T_{(2)}^{l o c, 1,+}=-G T_{(2)}^{l o c, 1,-} .
$$

This together with the sum formula (0.5) for the case $(h, p)=(1,+)$ yields

$$
G T_{(2)}^{l o c, 1, p}=(-1)^{p} 2 G T_{(2)}^{l o c, 0,+} .
$$

Using induction on genus $h$ together with (9.4) and (9.5) then shows 9.3). This completes the proof.

Remark 9.2. The proof of Proposition 9.1(b) by Kiem and Li (see Section 4 of KL1) proceeds as follows: they first obtained a sum formula similar to (9.2) using their sum formula and then showed (9.3) by calculating the local invariants $G T_{2}^{l o c, h, p}\left(\tau\left(F^{*}\right)\right)$ for all $h \geq 0$ using explicit algebro-geometric arguments.

\section{APPENDIX}

Let $G W_{d, g}(\cdot)$ and $G W_{\left(1^{d}\right),\left(1^{d}\right), g}(\cdot)$ respectively denote the absolute GW invariants of $\mathbb{P}_{0}$ for the class $d S$ with genus $g$ and the relative $G W$ invariants of $\mathbb{P}_{0}$ relative to distinct fibers $V_{1}$ and $V_{2}$ of $\mathbb{P}_{h}$ with contact constraint $C_{\left[V_{i}\right]^{d}}$ with $V_{i}$ (we will omit the fibers $V_{i}$ and the contact constraints $C_{\left[V_{i}\right]^{d}}$ in the notation). Since local 
invariants of spin curve of genus $h=0$ are $\mathrm{GW}$ invariants of $\mathbb{P}_{0}$, the lemma below shows formula (5.7) for the case when $h=0$ and $n \geq 3$.

Lemma 10.1. For $n \geq 3$, we have

$$
G W_{d, g}\left(\prod_{i=1}^{n} \tau_{k_{i}}\left(F^{*}\right)\right)=\frac{1}{(d !)^{2}} G W_{\left(1^{d}\right),\left(1^{d}\right), g}\left(\prod_{i=1}^{n} \phi_{i}^{k_{i}}\left(F^{*}\right)\right) .
$$

The proof consists of two steps.

Step 1. We will relate the descendent classes for GW invariants of $\mathbb{P}_{0}$ to the $\phi_{i}$ classes. Following [KM], we set

$$
\tau_{s_{i}} \phi_{i}^{t_{i}}\left(F^{*}\right)=\psi_{i}^{s_{i}} s t^{*} \phi_{i}^{t_{i}} \cup e v_{i}^{*}\left(F^{*}\right) .
$$

Lemma 10.2. Let $n \geq 3$. Then, for $s_{j} \geq 1$, we have

$$
\begin{aligned}
& G W_{d, g}\left(\prod_{i=1}^{n} \tau_{s_{i}} \phi_{i}^{t_{i}}\left(F^{*}\right)\right)=G W_{d, g}\left(\prod_{i=1}^{n} \tau_{s_{i}-\delta_{i j}} \phi_{i}^{t_{i}+\delta_{i j}}\left(F^{*}\right)\right) \\
& -\sum_{0<k<d} \delta_{k s_{j}}(-1)^{k-1} \frac{1}{k !} G W_{d-k, g}\left(\phi_{j}^{t_{j}}\left(F^{*}\right) \prod_{i \neq j} \tau_{s_{i}} \phi_{i}^{t_{i}}\left(F^{*}\right)\right) .
\end{aligned}
$$

Proof. It follows from Theorem 1.1 of $[\mathrm{KM}]$ that for $s_{j} \geq 1$,

$$
\begin{gathered}
G W_{d, g}\left(\prod_{i=1}^{n} \tau_{s_{i}} \phi_{i}^{t_{i}}\left(F^{*}\right)\right)=G W_{d, g}\left(\prod_{i=1}^{n} \tau_{s_{i}-\delta_{i j}} \phi_{i}^{t_{i}+\delta_{i j}}\left(F^{*}\right)\right), \\
+\sum_{a, 0<k \leq d} G W_{k, 0}\left(\tau_{s_{j}-1}\left(F^{*}\right) H^{a}\right) G W_{d-k, g}\left(\phi_{j}^{t_{j}}\left(H_{a}\right) \prod_{i \neq j} \tau_{s_{i}} \phi_{i}^{t_{i}}\left(F^{*}\right)\right),
\end{gathered}
$$

where $\left\{H_{a}\right\}$ and $\left\{H^{a}\right\}$ are Poincaré dual bases of $H^{*}\left(\mathbb{P}_{0}\right)$. Fix a basis $\left\{1, S^{*}+\right.$ $\left.F^{*}, F^{*}, \gamma^{*}\right\}$ and its dual basis $\left\{\gamma^{*}, F^{*}, S^{*}, 1\right\}$ of $H^{*}\left(\mathbb{P}_{0}\right)$, where $\gamma^{*}$ is the Poincaré dual of the point class of $\mathbb{P}_{0}$. Note that all degree zero $(d=0)$ invariants on the right-hand side of (10.3) vanish since $n \geq 3$; no degree zero maps can pass through two distinct fibers. Moreover, for any $d>0$ and $g$,

$$
\begin{gathered}
G W_{d, g}\left(\gamma^{*} \cdots\right)=0, \quad G W_{d, g}\left(\left(S^{*}+F^{*}\right) \cdots\right)=0, \\
G W_{d, g}\left(S^{*} \cdots\right)=-G W_{d, g}\left(F^{*} \cdots\right),
\end{gathered}
$$

where the first follows from $S^{2}=-1$, the second from $S(S+F)=0$ and the third from the second. Consequently, (10.3) becomes

$$
\begin{gathered}
G W_{d, g}\left(\prod_{i=1}^{n} \tau_{s_{i}} \phi_{i}^{t_{i}}\left(F^{*}\right)\right)=G W_{d, g}\left(\prod_{i=1}^{n} \tau_{s_{i}-\delta_{i j}} \phi_{i}^{t_{i}+\delta_{i j}}\left(F^{*}\right)\right) \\
-\sum_{0<k<d} G W_{k, 0}\left(\tau_{s_{j}-1}\left(F^{*}\right) F^{*}\right) G W_{d-k, g}\left(\phi_{j}^{t_{j}}\left(F^{*}\right) \prod_{i \neq j} \tau_{s_{i}} \phi_{i}^{t_{i}}\left(F^{*}\right)\right) .
\end{gathered}
$$

If $k \neq s_{j}$, then $G W_{k, 0}\left(\tau_{s_{j}-1}\left(F^{*}\right) F^{*}\right)=0$ by dimension count. So, it remains to show

$$
G W_{k, 0}\left(\tau_{k-1}\left(F^{*}\right) F^{*}\right)=(-1)^{k-1} / k !
$$

When $k=s_{j}$, the generalized Divisor Axiom (cf. Lemma 1.4 of [KM]) and (10.4) (applied to $G W_{k, 0}\left(\tau_{k-1}\left(F^{*}\right) F^{*} F^{*}\right)$ ) together with the fact that $\phi_{1}=0$ on $\overline{\mathcal{M}}_{0,3}$ 
and $G W_{1,0}\left(F^{*} F^{*}\right)=1$ give

$$
G W_{k, 0}\left(\tau_{k-1}\left(F^{*}\right) F^{*}\right)=\frac{1}{k} G W_{k, 0}\left(\tau_{k-1}\left(F^{*}\right) F^{*} F^{*}\right)=-\frac{1}{k} G W_{k-1,0}\left(\tau_{k-2}\left(F^{*}\right) F^{*}\right) .
$$

By induction, this shows (10.5), which completes the proof.

Step 2. We first show a formula for relative invariants that is analogous to (10.2) and then give a proof of Lemma 10.1. Recall that for the forgetful map $\pi_{\ell}: \overline{\mathcal{M}}_{g, n+\ell} \rightarrow$ $\overline{\mathcal{M}}_{g, n}$ that forgets the last $\ell$ marked points and for $1 \leq i \leq n$ we have

$$
\pi_{\ell}^{*} \phi_{i}=\phi_{i}-\sum \delta_{\{i\} \cup I},
$$

where the sum is over all $I \subset\{n+1, \ldots, n+\ell\}$ with $I \neq \emptyset$. For simplicity, we will write $\pi_{\ell}$ simply as $\pi$ when $\ell$ is even.

Lemma 10.3. Let $n \geq 3$. Then, for $s_{j} \geq 1$ we have

$$
\begin{gathered}
G W_{\left(1^{d}\right),\left(1^{d}\right), g}\left(\prod_{i=1}^{n} \phi_{i}^{s_{i}} \pi^{*} \phi_{i}^{t_{i}}\left(F^{*}\right)\right)=G W_{\left(1^{d}\right),\left(1^{d}\right), g}\left(\prod_{i=1}^{n} \phi_{i}^{s_{i}-\delta_{i j}} \pi^{*} \phi_{i}^{t_{i}+\delta_{i j}}\left(F^{*}\right)\right) \\
-\sum_{0<k<d} \delta_{k s_{j}}(-1)^{k-1} k !\left(\begin{array}{c}
d \\
k
\end{array}\right)^{2} G W_{\left(1^{d-k}\right),\left(1^{d-k}\right), g}\left(\pi^{*} \phi_{j}^{t_{j}}\left(F^{*}\right) \prod_{i \neq j} \phi_{i}^{s_{i}} \pi^{*} \phi_{i}^{t_{i}}\left(F^{*}\right)\right) .
\end{gathered}
$$

Proof. Without loss of generality, we may assume $j=1$. For the forgetful map $\pi=\pi_{2 d}$, let $\delta_{\{1\} \cup I}$ be a class as in (10.6) and denote by $\overline{\mathcal{M}}\left(\delta_{\{1\} \cup I}\right)$ the boundary stratum of $\overline{\mathcal{M}}_{g, n+2 d}$ whose fundamental class is Poincaré dual to $\delta_{\{1\} \cup I}$. Then for $m=|I|$ there is a gluing map

$$
\eta_{\sigma}: \overline{\mathcal{M}}_{0, m+2} \times \overline{\mathcal{M}}_{g, n+2 d-m} \rightarrow \overline{\mathcal{M}}_{g, n+2 d}
$$

whose image is $\overline{\mathcal{M}}\left(\delta_{\{1\} \cup I}\right)$. This gluing map is obtained by identifying the second marked point of the first component with the first marked point of the second component. We have

$$
\eta_{\sigma}^{*}\left(\phi_{1}\right)=\phi_{1} \otimes 1 \quad \text { and } \quad \eta_{\sigma}^{*} \circ \pi^{*}\left(\phi_{1}\right)=1 \otimes \pi_{2 d-m}^{*}\left(\phi_{1}\right),
$$

where the first equality follows from Lemma $1.2(\mathrm{~b})$ and the second from the fact that under the composition map $\pi \circ \eta_{\sigma}$ the first component collapses to a point.

Choose two distinct fibers $V_{1}$ and $V_{2}$ of $\mathbb{P}_{0}$, and, for simplicity, we set

$$
\mathcal{M}^{V}=\mathcal{M}_{g, n,\left(1^{d}\right),\left(1^{d}\right)}^{V_{1}, V_{2}}\left(\mathbb{P}_{0}, d S\right) \quad \text { and } \quad \Phi=\phi_{1}^{s_{1}-1} \pi^{*} \phi_{1}^{t_{1}} \prod_{i>1} \phi_{i}^{s_{i}} \pi^{*} \phi_{i}^{t_{i}}
$$

where $V=V_{1} \sqcup V_{2}$. Let $G$ be a geometric representative of the Poincaré dual of the pull-back class $\eta_{\sigma}^{*} \Phi$. One can then choose a (smooth) family of geometric representatives $G_{t}$ of the Poincaré dual of the class $\delta_{\{1\} \cup I} \cup \Phi$ with $G_{0}=\eta_{\sigma}(G)$. Let $B$ be a product of $n$ distinct generic fibers $B_{i}$ of $\mathbb{P}_{0}$, each of which is disjoint with $V$.

Suppose $\mathcal{M}^{V} \cap B \cap G_{t} \neq \emptyset$ for all small $t$. Then, by the Gromov Compactness Theorem, after passing to subsequences, as $t \rightarrow 0$ every sequence $f_{t} \in \mathcal{M}^{V} \cap B \cap G_{t}$ converges to

$$
(f, C) \in C \mathcal{M}^{V} \cap B \cap \eta_{\sigma}(G),
$$

where $C \mathcal{M}^{V}$ is the closure of $\mathcal{M}^{V}$ in $\overline{\mathcal{M}}_{g, n+2 d}\left(\mathbb{P}_{0}, d S\right)$. The closure $C \mathcal{M}^{V}$ has a stratification in which each stratum consisting of maps with domains with more than one node has dimension at least 4 less than $2 \operatorname{deg}(\Phi)+2+2 n$ (cf. Lemma 7.6 of [IP2]). Thus the domain $C \in \overline{\mathcal{M}}\left(\delta_{I}\right)$ of $f$ has one node by dimension count. The 
limit map $f$ splits as $f=\left(f_{1}, f_{2}\right)$ such that each $f_{i}$ is $V$-regular unless it represents the trivial homology class. In our case, both $f_{1}$ and $f_{2}$ are $V$-regular maps since the image of $f_{1}$ passes through $V$ and $B_{1}$ and since the image of $f_{2}$ passes through $(n-1)>2$ distinct fibers $B_{i}$ where $2 \leq i \leq n-1$. For some $0<k<d$, we have

- $f_{1}$ (resp. $\left.f_{2}\right)$ has contact vector $\left(1^{k}\right)$ (resp. $\left.\left(1^{d-k}\right)\right)$ with both $V_{1}$ and $V_{2}$, and hence

- $f \in e v_{\sigma}^{-1}(\triangle) \cap B \cap \eta(G)$ (under the natural inclusion $e v_{\sigma}^{-1}(\triangle) \hookrightarrow \mathcal{M}^{V}$ ),

where $\triangle$ is the diagonal of $\mathbb{P}_{0} \times \mathbb{P}_{0}$ and $e v_{\sigma}$ is the evaluation map

$e v_{\sigma}=e v_{2} \times e v_{1}: \mathcal{M}_{0,2,\left(1^{k}\right),\left(1^{k}\right)}^{V_{1}, V_{2}}\left(\mathbb{P}_{0}, k S\right) \times \mathcal{M}_{g, n,\left(1^{d-k}\right),\left(1^{d-k}\right)}^{V_{1}, V_{2}}\left(\mathbb{P}_{0},(d-k) S\right) \mapsto \mathbb{P}_{0} \times \mathbb{P}_{0}$

On the other hand, the condition of contact order $\left(1^{d}\right)$ with $V$ is an open condition. The Gluing Theorem of RT2 thus implies that for each small $t$ one can uniquely smooth $f$ (at a node) to produce a $V$-regular map in $\mathcal{M}^{V} \cap G_{t}$. Consequently, we have

$$
\left[e v_{\sigma}^{-1}(\triangle)\right] \cap \eta_{\sigma}^{*}(\Phi) \otimes\left(F^{*}\right)^{n}=\left[\mathcal{M}^{V}\right] \cap\left(\delta_{\{1\} \cup I} \cup \Phi\right) \otimes\left(F^{*}\right)^{n} .
$$

It follows from (5.6), (10.7), (10.8) and the splitting of the diagonal $\triangle$ as in the proof of Lemma 10.2 (see the paragraph above (10.4)) that

$$
\begin{aligned}
G W_{\left(1^{d}\right),\left(1^{d}\right), g}\left(\prod_{i=1}^{n} \phi_{i}^{s_{i}} \pi^{*} \phi_{i}^{t_{i}}\left(F^{*}\right)\right) & =G W_{\left(1^{d}\right),\left(1^{d}\right), g}\left(\prod_{i=1}^{n} \phi_{i}^{s_{i}-\delta_{i 1}} \pi^{*} \phi_{i}^{t_{i}+\delta_{i 1}}\left(F^{*}\right)\right) \\
& -\sum_{0<k<d}\left(\begin{array}{c}
d \\
k
\end{array}\right)^{2} G W_{\left(1^{k}\right),\left(1^{k}\right), 0}\left(\phi_{1}^{s_{1}-1}\left(F^{*}\right) F^{*}\right) \\
& \cdot G W_{\left(1^{d-k}\right),\left(1^{d-k}\right), g}\left(\pi^{*} \phi_{1}^{t_{1}}\left(F^{*}\right) \prod_{i>1} \phi_{i}^{s_{i}} \pi^{*} \phi_{i}^{t_{i}}\left(F^{*}\right)\right),
\end{aligned}
$$

where the factor $\left(\begin{array}{c}d \\ k\end{array}\right)^{2}$ reflects the fact that the classes $\delta_{\{1\} \cup I}$ in (10.8) are obtained by choosing $k$ contact points with $V_{1}$ and $k$ contact points with $V_{2}$. Observe that by dimension if $k \neq s_{1}$, then $G W_{\left(1^{k}\right),\left(1^{k}\right), 0}\left(\phi_{1}^{s_{1}-1}\left(F^{*}\right) F^{*}\right)=0$. Thus, it remains to show that

$$
G W_{\left(1^{k}\right),\left(1^{k}\right), 0}\left(\phi^{k-1}\left(F^{*}\right) F^{*}\right)=(-1)^{k-1} k ! .
$$

The sum of formula (4.15) for $h=0$ gives

$$
\begin{aligned}
G W_{\left(1^{k}\right),\left(1^{k}\right), 0}\left(\phi^{k-1}\left(F^{*}\right) F^{*} F^{*}\right) & =\frac{1}{k !} G W_{\left(1^{k}\right),\left(1^{k}\right), 0}\left(\phi^{k-1}\left(F^{*}\right) F^{*}\right) \cdot G T_{\left(1^{k}\right),\left(1^{k}\right)}^{\mathbb{F}_{0}}\left(F^{*}\right) \\
& =k G W_{\left(1^{k}\right),\left(1^{k}\right), 0}\left(\phi^{k-1}\left(F^{*}\right) F^{*}\right)
\end{aligned}
$$

where the second equality follows from (3.8) and the Divisor Axiom. On the other hand, together with the facts $\phi=0$ on $\overline{\mathcal{M}}_{0,3}$ and $G W_{(1),(1), 0}\left(F^{*} F^{*}\right)=1$, formula (10.9) shows that

$$
G W_{\left(1^{k}\right),\left(1^{k}\right), 0}\left(\phi^{k-1}\left(F^{*}\right) F^{*} F^{*}\right)=-k^{2} G W_{\left(1^{k-1}\right),\left(1^{k-1}\right), 0}\left(\phi^{k-2}\left(F^{*}\right) F^{*}\right) .
$$

By induction, (10.11) and (10.12) thus imply (10.10). This completes the proof. 
Proof of Lemma 10.1. It suffice to show that for $n \geq 3$,

$$
G W_{d, g}\left(\prod_{i=1}^{n} \tau_{s_{i}} \phi_{i}^{t_{i}}\left(F^{*}\right)\right)=\frac{1}{(d !)^{2}} G W_{\left(1^{d}\right),\left(1^{d}\right), g}\left(\prod_{i=1}^{n} \phi_{i}^{s_{i}} \pi^{*} \phi_{i}^{t_{i}}\left(F^{*}\right)\right) .
$$

When $\sum s_{i}=0$, 10.13) follows from Lemma 5.2. Suppose that 10.13) holds for any $d, g$ and $n \geq 3$ whenever $\sum s_{i}<\ell$. Then, Lemma 10.2 and Lemma 10.3 show that (10.13) also holds when $\sum s_{i}=\ell$. Therefore, 10.13) follows from induction on the $\operatorname{sum} \sum s_{i}$.

\section{ACKNOWLEDGMENTS}

The author is very thankful to Thomas H. Parker for valuable discussions and to the referees for their corrections and useful comments. The author also thanks Bumsig Kim and Young-Hoon Kiem for discussions and Rahul Pandharipande, Davesh Maulik and Aleksey Zinger for their interest in this work.

\section{REFERENCES}

[ACV] D. Abramovich, A. Corti, and A. Vistoli, Twisted bundles and admissible covers, Commun. in Algebra. 31 (2003), 3547-3618. MR2007376 (2005b:14049)

[AC] E. Arbarello and M. Cornalba, Calculating cohomology groups of moduli spaces of curves via algebraic geometry, Inst. Hautes Études Sci. Publ. Math. No. 88 (1998), 97-127. MR:1733327(2001h:14030)

[C] M. Cornalba, Moduli of curves and theta charateristics, Lectures on Riemann Surfaces, 560-589, World Scientific, Singapore 1989. MR1082361 (91m:14037)

[FP] C. Faber and R. Pandharipande, Hodge integrals and Gromov-Witten theory, Invent. Math. 139 (2000), no. 1, 173-199. MR1728879 (2000m:14057)

[HM] J. Harris and I. Morrison, Moduli of curves, Graduate Texts in Mathematics, 187. SpringerVerlag, New York, 1998. MR1631825 (99g:14031)

[IP1] E. Ionel and T.H. Parker, Relative Gromov-Witten invariants, Annals of Math. 157 (2003), 45-96. MR1954264(2004a:53112)

[IP2] E. Ionel and T.H. Parker, The symplectic sum formula for Gromov-Witten invariants, Annals of Math. 159 (2004), 935-1025. MR2113018 (2006b:53110)

[KL1] Y-H. Kiem and J. Li, Gromov-Witten invariants of varieties with holomorphic 2-forms, preprint, math.AG/07072986.

[KL2] Y-H. Kiem and J. Li, Low degree GW invariants of spin surfaces, Pure Appl. Math. Q. 7 (2011), no. 4, 1449-1476. MR2918169

[KL3] Y-H. Kiem and J. Li, Low degree GW invariants of surfaces II, Science China Math. 54 (2011), no. 8, 1679-1706. MR.2824966

[KM] M. Kontsevich and Y.I. Manin, Relations between the correlators of the topological sigma model coupled to gravity, Commun. Math. Phys. 196 (1998), 385-398. MR1645019 (99k:14040)

[L] J. Lee, Family Gromov-Witten invariants for Kähler surfaces, Duke Math. J. 123 (2004), no 1, 209-233. MR2060027(2005d:53141)

[Lo] E. Looijenga, Smooth Deligne-Mumford compactifications by means of Prym level structures, J. Algebraic Geom. 3 (1994), no. 2, 283-29. MR.1257324 (94m:14029)

[LP1] J. Lee and T.H. Parker, A Structure Theorem for the Gromov-Witten Invariants of Kähler Surfaces, J. Diff. Geom. 77 (2007), 483-513. MR2362322 (2010b:53159)

[LP2] J. Lee and T.H. Parker, An obstruction bundle relating Gromov-Witten invariants of curves and Kähler surfaces, preprint, arXiv:0909.3610.

[LT] J. Li and G. Tian, Virtual moduli cycles and Gromov-Witten invariants of general symplectic manifolds, Topics in symplectic 4-manifolds (Irvine, CA, 1996), 47-83, First Int. Press Lect. Ser., I, Internat. Press, Cambridge, MA, 1998. MR.1635695 (2000d:53137)

[MP] D. Maulik and R. Pandharipande, New calculations in Gromov-Witten theory, Pure Appl. Math. Q. 4 (2008), no. 2, part 1, 469-500. MR2400883(2009d:14073) 
[RT1] Y. Ruan and G. Tian, A mathematical theory of quantum cohomology, J. Differential Geom. 42 (1995), 259-367. MR1366548 (96m:58033)

[RT2] Y. Ruan and G. Tian, Higher genus symplectic invariants and sigma models coupled with gravity, Invent. Math. 130 (1997), no. 3, 455-516. MR1483992 (99d:58030)

[T] L. Tu, Hodge theory and the local Torelli problem, Mem. Amer. Math. Soc. 43 (1983), no. 279. MR699239 (84k:14008)

Department of Mathematics, University of Central Florida, Orlando, Florida 32816

E-mail address: junlee@mail.ucf.edu 